\title{
Aberrations in Notch-Hedgehog signalling reveal cancer stem cells harbouring conserved oncogenic properties associated with hypoxia and immunoevasion
}

\author{
Wai Hoong Chang ${ }^{1}$ and Alvina G. Lai ${ }^{1}$
}

\begin{abstract}
BACKGROUND: Cancer stem cells (CSCs) have innate abilities to resist even the harshest of therapies. To eradicate CSCs, parallels can be drawn from signalling modules that orchestrate pluripotency. Notch-Hedgehog hyperactivation are seen in CSCs, yet, not much is known about their conserved roles in tumour progression across cancers.

METHODS: Employing a comparative approach involving 21 cancers, we uncovered clinically-relevant, pan-cancer drivers of Notch and Hedgehog. GISTIC datasets were used to evaluate copy number alterations. Receiver operating characteristic and Cox regression were employed for survival analyses.

RESULTS: We identified a Notch-Hedgehog signature of 13 genes exhibiting high frequencies of somatic amplifications leading to transcript overexpression. The signature successfully predicted patients at risk of death in five cancers $(n=2278)$ : glioma $(P<$ $0.0001)$, clear cell renal cell $(P=0.0022)$, papillary renal cell $(P=0.00099)$, liver $(P=0.014)$ and stomach $(P=0.011)$. The signature was independent of other clinicopathological parameters and offered an additional resolution to stratify similarly-staged tumours. High-risk patients exhibited features of stemness and had more hypoxic tumours, suggesting that hypoxia may influence CSC behaviour. Notch-Hedgehog ${ }^{+}$CSCs had an immune privileged phenotype associated with increased regulatory T cell function. CONCLUSION: This study will set the stage for exploring adjuvant therapy targeting the Notch-Hedgehog axis to help optimise therapeutic regimes leading to successful CSC elimination.
\end{abstract}

British Journal of Cancer (2019) 121:666-678; https://doi.org/10.1038/s41416-019-0572-9

\section{BACKGROUND}

Tumours are far from homogeneous masses, yet many contemporary therapies continue to treat them as such. It has become increasingly clear that a minor population of tumour cells known as cancer stem cells (CSCs) contribute to treatment resistance as they have the propensity to tolerate DNA damage ${ }^{1,2}$ and evade immune detection $^{3}$ to give rise to new tumours post therapy. Identification of CSCs has remained a challenging endeavour since they only make up a small proportion of the tumour and are histologically similar to non-stem cancer cells. Moreover, molecular markers that identify CSCs are often cancer-type dependent, which limit their broad scale applications. ${ }^{4}$ CSCs share many qualities with embryonic or adult stem cells. For example, activation of signalling pathways involved in coordinating cellular homoeostasis, morphogenesis and cell fate determination (TGF- $\beta$, Wnt, Notch and Hedgehog) are often seen in CSCs. These pathways rarely act in isolation and significant crosstalk between them have been reported. ${ }^{5}$

In order to fully exploit these pathways for CSC therapy, pancancer explorations are warranted to reveal conserved components that can be prioritised as therapeutic targets. Concentrating on Notch and Hedgehog signalling pathways, we seek to attain a comprehensive understanding of how somatic copy number alterations and expression profiles of pathway genes along with their downstream targets could influence tumour progression and prognosis. The role of Notch signalling in oncogenesis was initially discovered in T cell acute lymphoblastic leukaemia. ${ }^{6}$ Since then, multiple studies on Notch signalling have demonstrated both oncogenic and tumour suppressive functions in haematological and solid malignancies, implying its pleiotropic nature that is very much dependent on cellular types. ${ }^{7}$ Hedgehog is a morphogen that regulates a signalling cascade involving the Smoothened protein to influence morphogenetic processes such as proliferation and differentiation. ${ }^{8}$ Interactions between Notch and Hedgehog signalling have been demonstrated in multiple cancers. Hes 1 , a Notch effector, is targeted by sonic hedgehog in neural cells. ${ }^{9}$ When Patched, a negative regulator of Hedgehog, is abrogated in mice, this gives rise to medulloblastoma with enhanced Notch signalling. ${ }^{10}$ Hedgehog signalling promotes the expression of Jagged 2 (a Notch ligand) ${ }^{11}$ and in ovarian cancer mice models, inhibition of Jagged 1 would sensitise tumours to docetaxel treatment by affecting GLI2 function. ${ }^{12}$ Concurrent activation of Hedgehog and Notch signalling was observed in prostate cancer cells that were resistant to docetaxel. ${ }^{13}$ Glioblastoma treated with a Notch inhibitor was subsequently desensitised to further Notch suppression as they upregulate Hedgehog signalling. ${ }^{14}$

These studies highlight the importance of Notch-Hedgehog interactions in cancer, which calls for a better understanding of

${ }^{1}$ Institute of Health Informatics, University College London, 222 Euston Road, London NW1 2DA, UK

Correspondence: Alvina G. Lai (alvina.lai@ucl.ac.uk)

Received: 21 January 2019 Revised: 13 August 2019 Accepted: 20 August 2019

Published online: 16 September 2019 
their relationship and also to reveal crosstalk with other pathways involved in regulating CSC function. Harnessing genomic and transcriptomic sequences of 21 cancer types, we performed a comprehensive investigation linking genomic alterations to transcriptional dysregulation of Notch-Hedgehog pathway genes. We discovered conserved patterns of Notch-Hedgehog hyperactivation across cancers and revealed putative driver genes that were associated with CSC phenotypes underpinning poor clinical outcomes. We also examined the relationship between the tumour microenvironment (hypoxia and immune suppression) and Notch-Hedgehog ${ }^{+}$CSCs. In-depth knowledge of the NotchHedgehog signalling axis afforded by this study will set the stage for exploring combinatorial chemotherapy targeting both pathways simultaneously to potentially eradicate CSCs.

\section{MATERIALS AND METHODS}

A total of 72 genes associated with Notch and Hedgehog signalling were retrieved from the Kyoto Encyclopedia of Genes and Genomes (KEGG) database listed in Table S1.

\section{Study cohorts}

We retrieved transcriptomic and genomic profiles of 21 cancer types $(n=18,484)$ including their non-tumour counterparts from The Cancer Genome Atlas and Broad Institute GDAC Firehose ${ }^{15}$ (Table S2).

Somatic copy number alterations analyses

We retrieved Firehose Level 4 copy number variation datasets in the form of GISTIC gene-level tables, which provided discrete amplification and deletion indicators. ${ }^{16}$ A sample was defined as 'deep amplification' for values that were higher than the maximum median copy-ratio for each chromosome arm $(+2)$. Samples with values less than the minimum median copy-ratio for each chromosome arm were called 'deep deletions' (-2). GISTIC indicators of +1 and -1 represented shallow amplifications and deletions respectively.

Calculating Notch-Hedgehog 13-gene scores, hypoxia scores and regulatory $\mathrm{T}$ cell (Treg) scores

The Notch-Hedgehog 13-gene signature was employed to calculate a score for each patient. It comprised of the following genes: $J A G 1$, LFNG, DTX2, DLL3, GPR161, PSENEN, GLI1, HES1, PTCRA, DTX3L, ADAM17, KIF7 and NOTCH1. Hypoxia scores were calculated from 52hypoxia signature genes. ${ }^{17}$ Treg scores were calculated based on the overlap between four Treg signatures, ${ }^{18-21}$ consisting of 31 genes: FOXP3, TNFRSF18, TNFRSF9, TIGIT, IKZF2, CTLA4, CCR8, TNFRSF4, IL2RA, BATF, IL2RB, CTSC, CD27, PTTG1, ICOS, CD7, TFRC, ERI1, GLRX, NCF4, PARK7, HTATIP2, FCRL3, CALM3, DPYSL2, CSF2RB, CSF1, IL1R2, VDR, $A C P 5$ and MAGEH1. Scores were calculated from the average $\log _{2}$ expression values of 13,52 or 31 genes representing NotchHedgehog, hypoxia and Tregs respectively. Kaplan-Meier analyses of the Notch-Hedgehog signature were performed on patients separated into quartiles based on their 13-gene scores. For analyses in Figs. 4-6, patients were separated into four groups using median 13-gene scores and median CSC transcription factor expression levels (EZH2, REST and SUZ12), hypoxia scores or Treg scores as thresholds for Kaplan-Meier and Cox regression analyses. Nonparametric Spearman's rank-order correlation tests were used to investigate the relationship between 13-gene scores and TF expression levels, hypoxia scores or Treg scores.

Multidimensional scaling, differential expression and survival analyses

As per the journal's guidelines, we have not repeated methods here as we have previously published detail methods for multidimensional scaling (MDS), differential expression and survival analyses. ${ }^{22-24}$ Briefly, MDS analysis was employed to visualise samples' distance (tumour and non-tumour) in reduced 2-dimensional space. The $R$ vegan package was employed for MDS ordination using Euclidean distances. Permutational multivariate analysis of variance (PERMANOVA) was used to investigate statistical differences between tumour and non-tumour samples. The linear model and Bayes method were employed for differential expression analyses, followed by the BenjaminiHochberg false discovery rate method. Kaplan-Meier, Cox proportional hazards and receiver operating characteristic survival analyses were performed using $\mathrm{R}$ survminer, survival and survcomp packages.

Functional enrichment and transcription factor (TF) analyses Differential expression analyses as mentioned previously were performed on patients separated into quartiles 4 and 1 based on their 13-gene scores. Differentially expressed genes were mapped against KEGG and Gene Ontology (GO) databases using GeneCodis $^{25}$ to determine pathways that were enriched. The Enrichr tool was used to determine whether differentially expressed genes were enriched for stem cell TFs binding targets by comparing chromatin immunoprecipitation sequencing profiles from ChEA and ENCODE databases. ${ }^{26}$

The $\mathrm{R}$ ggplot2 and pheatmap packages were used to generate all plots.

\section{RESULTS}

Recurrently amplified driver genes associated with Notch and Hedgehog activation in 21 diverse cancer types

To characterise the extent of Notch and Hedgehog signalling and identify common molecular subtypes, we examined somatic copy number alterations (SCNAs) and differential expression (tumour versus non-tumour) patterns of 72 genes in 18,484 cases of clinically annotated stage I to IV samples representing 21 cancer types (Fig. 1a; Tables S1 and S2). We found that 70 out of 72 genes were recurrently amplified in at least $20 \%$ of samples per cancer type in at least one cancer type (Fig. 1a). Lung squamous cell carcinoma (LUSC) had the highest fraction of samples harbouring amplified Hedgehog genes, while endometrial cancer (UCEC) had the fewest somatic gains (Fig. 1b). When considering Notch gene amplifications, LUSC also emerged as the top candidate while clear cell renal cell carcinoma (KIRC) had the fewest number of Notch gene amplifications (Fig. 1b). In terms of focal deletions, this was also the highest in LUSC for Hedgehog genes and renal chromophobe carcinoma (KICH) for Notch genes (Fig. 1b).

Focusing on recurrently amplified genes, we identified 35 genes (Hedgehog pathway: 13 genes; Notch pathway: 22 genes) that were gained in $>20 \%$ of samples and in at least one-third of cancer types (>7 cancers) (Fig. 1c). GLI3, SMURF1, RBPJL, JAG1, LFNG and DTX2 were some of the most amplified genes present in at least 18 cancers (Fig. 1c). In contrast, KIF7, NOTCH1, MAML and ADAM17 were the least amplified genes (Fig. 1c). LUSC had the highest number of amplified genes ( 34 genes) followed by 33 genes in oesophageal carcinoma (ESCA) and stomach and oesophageal carcinoma (STES) and 32 genes in stomach adenocarcinoma (STAD) and bladder urothelial carcinoma (BLCA) (Fig. 1C). In contrast, only 8 genes were amplified in UCEC (Fig. 1C).

SCNA events associated with overexpression could represent candidate driver genes since positive correlations between gene amplification and overexpression are indicative of a gain-offunction. ${ }^{27}$ Differential expression analyses between tumour and adjacent non-tumour samples revealed that 13 of the amplified genes were also significantly upregulated (>1.5-fold-change, $P<0.05$ ) in tumours of at least 7 cancer types (Fig. 1c). These genes were prioritised as a Notch-Hedgehog signature potentially representative of multiple cancers: JAG1, LFNG, DTX2, DLL3, GPR161, PSENEN, GLI1, HES1, PTCRA, DTX3L, ADAM17, KIF7 and NOTCH1 (Fig. 1C). 


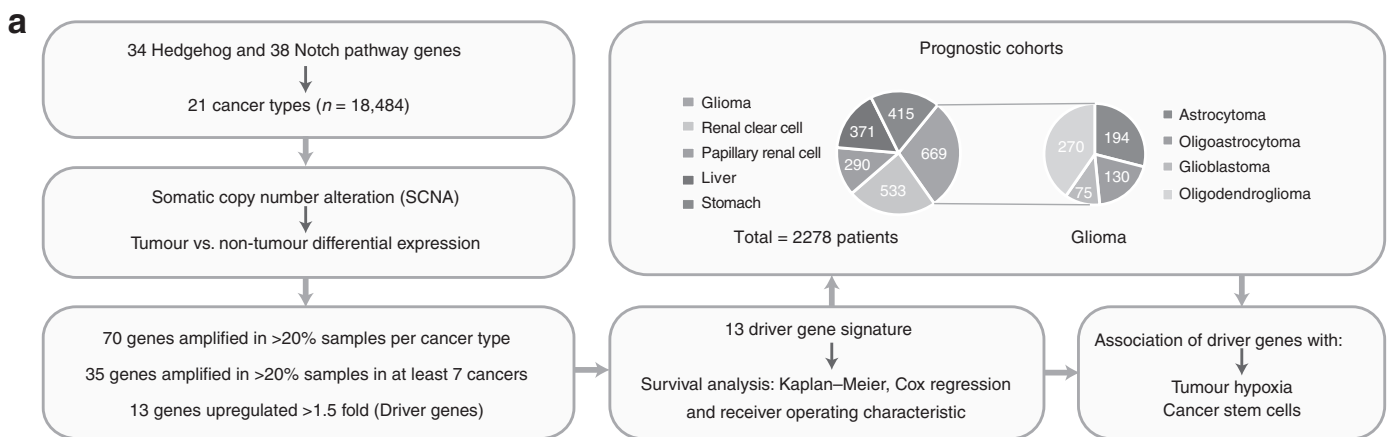

b
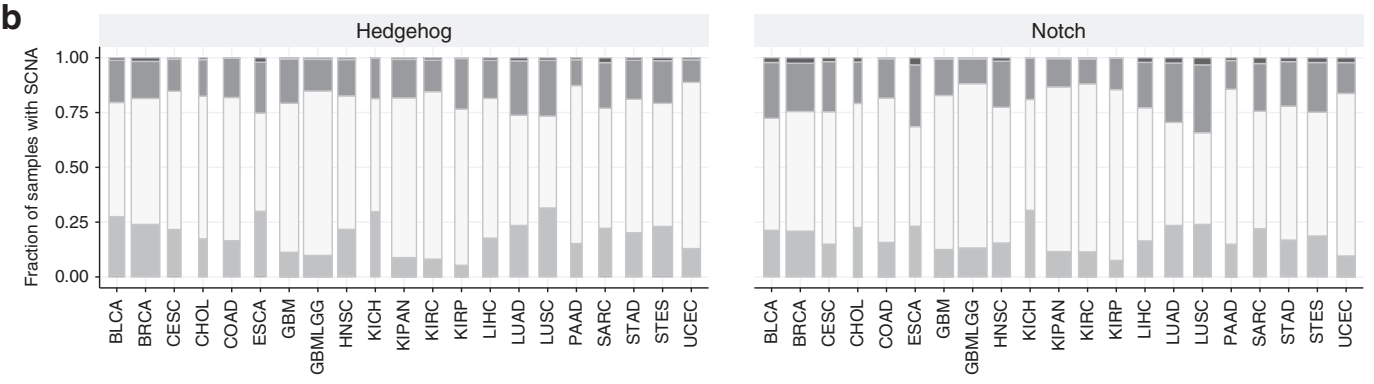

Deep amplification

Shallow amplification

No alteration Shallow deletion

C
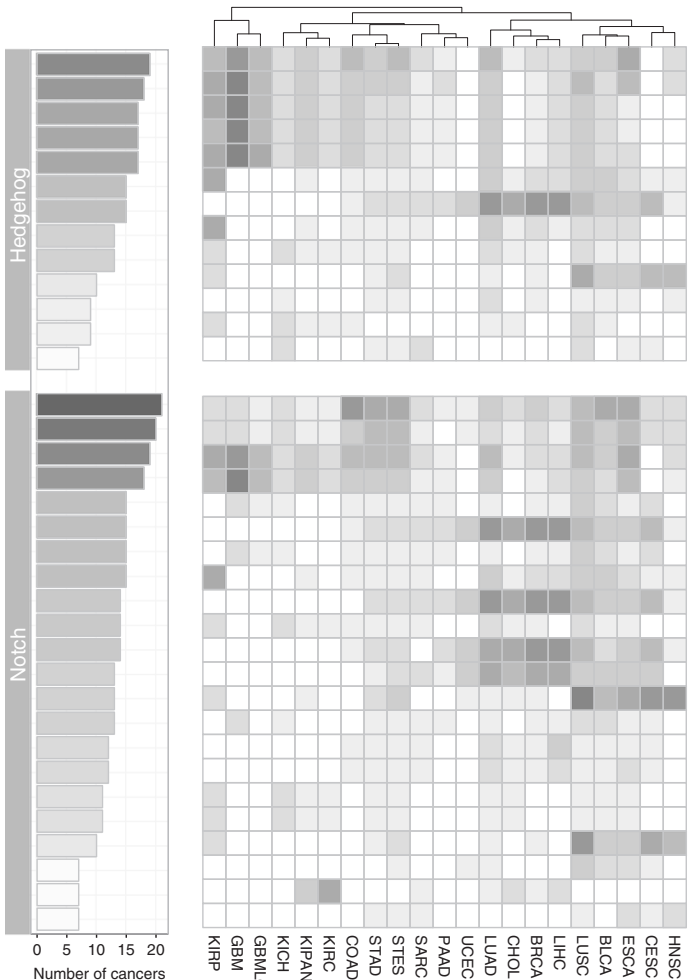

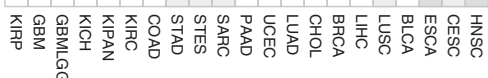

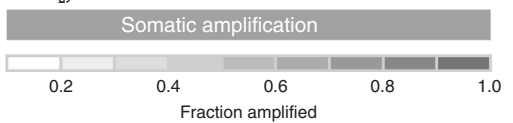

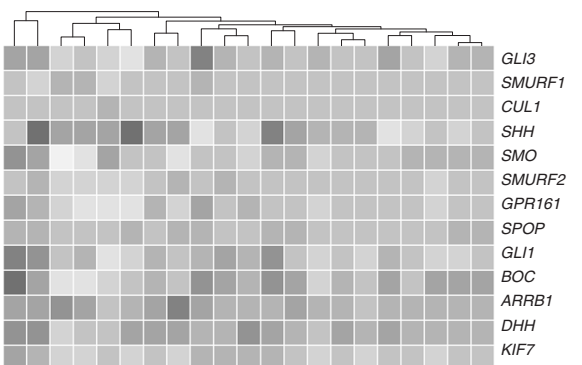

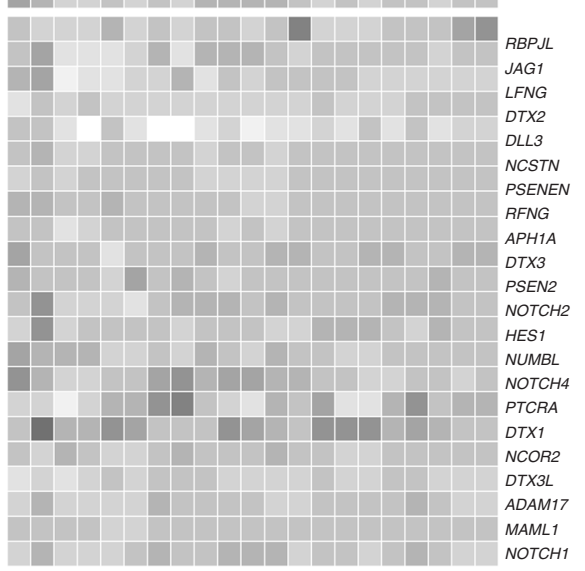

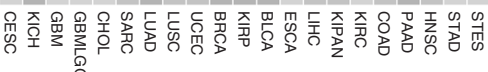

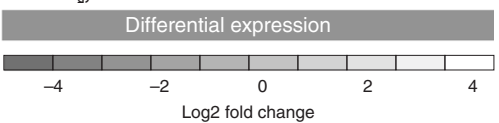

A 13-gene Notch-Hedgehog signature predicts survival outcomes in five cancers

Tumours displayed various degrees of somatic gains and overexpression of Notch-Hedgehog pathway genes (Fig. 1), suggesting that aberrant activation of these pathways may influence disease progression and survival outcomes. We employed univariate Cox proportional hazards regression analyses to test the prognostic roles of individual Notch-Hedgehog signature genes across 20 cancer types where survival data is available. Prognosis appeared to tissue type-dependent (Fig. S1). All 13 genes were prognostic in the glioma dataset (GBMLGG), consisting of samples from patients with astrocytoma, oligoastrocytoma, oligodendroglioma and 
Fig. 1 Pan-cancer drivers of Notch-Hedgehog signalling. a Schematic diagram illustrating the study design and the identification of NotchHedgehog driver genes, which represent the 13-gene signature. SCNA and expression profiles of 72 Notch-Hedgehog pathway genes were interrogated in 21 cancer types involving 18,484 patients. We identified 70 genes as amplified in at least $20 \%$ of samples and 35 genes that were amplified in at least $20 \%$ of samples in at least 7 cancer types. Differential expression analyses between tumour and non-tumour samples revealed that the 13 recurrently amplified genes were also upregulated, potentially indicating a gain-of-function. These 13 genes were prioritised as a Notch-Hedgehog signature, which was prognostic in five cancer types involving 2278 patients. Associations of the signature with tumour microenvironmental features of hypoxia and immunity were also investigated. Pie slices indicate the number of samples within each cancer type. b Stacked bar graphs represent the proportion of samples in each cancer type with SCNA of Hedgehog and Notch pathway genes. Width of the bars reflect the number of samples within each cancer. c Somatic gains and differential expression profiles of 35 NotchHedgehog genes that were recurrently amplified in at least 7 cancer types (one-third of all cancers). Cumulative bar charts on the left represent the number of cancers with at least $20 \%$ of samples with somatic amplification. Heatmap on the left represents the extent of somatic gains for each of the 35 genes separated into Hedgehog and Notch signalling pathways across 21 cancers. Heatmap intensities depict the fraction of the cohort in which a given gene is amplified. Columns (cancer types) were ordered using Euclidean distance metric and hierarchical clustering to reveal cancers that were similar. Heatmap on the right represents tumour and non-tumour differential expression values $\left(\log _{2}\right)$ for the 35 genes. Genes highlighted in red represent the 13 Notch-Hedgehog signature genes. Cancer abbreviations were listed in Table S2

glioblastoma multiforme (Fig. S1). A majority of the genes (9 out of 13) were associated with poor prognosis (hazard ratio $[H R]>1$, $P<0.05$ ) (Fig. S1). However, despite showing high frequencies of SCNAs (Fig. 1c), none of the 13 genes harboured prognostic information in patients with LUSC, cholangiocarcinoma (CHOL) or oesophageal carcinoma (ESCA) (Fig. S1).

We next considered all 13 genes as a group in assessing prognosis. For each patient, we calculated their 13-gene scores by taking the average expression of all genes. Patients were separated into survival quartiles based on their 13-gene scores. Remarkably, Kaplan-Meier estimates and log-rank tests revealed that the 13-gene signature accurately predicted patients at higher risk of death in five cancer types $(n=2278)$ : glioma $(P<0.0001)$, clear cell renal cell $(P=0.0022)$, papillary renal cell $(P=0.00099)$, liver $(P=0.014)$ and stomach $(P=0.011)$ (Fig. 2a). Patients within the 4th quartile had significantly poorer survival rates compared to those within the 1st quartile: glioma $(\mathrm{HR}=3.386, P<0.0001)$, clear cell renal cell $(\mathrm{HR}=2.177, P=0.00048)$, papillary renal cell $(\mathrm{HR}=4.881, P=0.0053)$, liver $(\mathrm{HR}=2.627, P=0.0039)$ and stomach ( $\mathrm{HR}=2.217, P=0.014)$ (Table S3). When comparing tumour and non-tumour expression patterns, Mann-Whitney-Wilcoxon tests revealed that a vast majority of the 13 genes were significantly upregulated in tumours of these cancers (Fig. S2) where hyperactivation of Notch-Hedgehog signalling was associated with adverse survival outcomes (Fig. 2a). Multidimensional scaling analyses revealed that the 13 genes could accurately distinguish tumour from non-tumour samples in these cancers (Fig. 2b), suggesting that Notch-Hedgehog transcriptional states could be used to identify cells with oncogenic properties.

Multivariate Cox proportional hazards regression was used to determine whether the signature was confounded by other clinicopathological features. Tumour, node, metastasis (TNM) staging is frequently used for patient stratification. Even after accounting for TNM staging, the signature remained an independent predictor of survival: clear cell renal cell $(H R=1.731, P=$ $0.014)$, papillary renal cell $(H R=2.297, P=0.042)$, liver $(H R=$ 2.146; $P=0.024)$ and stomach (HR=2.161, $P=0.017)$ (Table S3). Given that both the signature and tumour stage were independent of each other, we reason that the signature could be used to improve TNM staging. We observed that Notch-Hedgehog driver genes offered an additional resolution in tumour classification for further stratification of similarly-staged tumours in these cancers: clear cell renal cell $(P<0.0001)$, papillary renal cell $(P<0.0001)$, liver $(P<0.0001)$ and stomach $(P=0.0068)$ (Fig. 3a).

Glioma samples are classified into four histological categories with varying severity: low-grade astrocytoma, low-grade oligodendroglioma, low-grade oligoastrocytoma (consisting of both abnormal astrocytoma and oligodendroglioma cells), and grade IV glioblastoma multiforme. Kaplan-Meier analyses of glioma samples grouped by histology revealed that the signature remained prognostic in astrocytoma $(P=0.038)$, oligoastrocytoma $(P=$ $0.0018)$ and glioblastoma multiforme $(P=0.045)$ (Fig. 3b). Patients with low-grade gliomas stratified by the signature into the $4^{\text {th }}$ quartile had significantly higher death risks compared to those within the 1st quartile: astrocytoma $(H R=2.535, P=0.021)$, oligoastrocytoma (HR=4.169, $P=0.014)$ and glioblastoma multiforme ( $\mathrm{HR}=2.163, P=0.042$ ) (Table S3).

To evaluate the predictive performance of the signature, we employed receiver operating characteristic (ROC) analyses and compared area under the curves (AUCs) derived from the signature versus those derived from TNM staging. The signature had greater sensitivity and specificity in predicting 5-year overall survival compared to TNM staging: papillary renal cell (AUC = 0.796 vs. AUC 0.640) and stomach (AUC $=0.710$ vs. $A U C=0.561$ ) (Fig. 3c). Importantly, when used as a combined model with TNM staging, it outperformed either the signature or TNM when considered alone, suggesting that incorporating molecular subtype information on Notch-Hedgehog signalling allowed more precise stratification: clear cell renal cell $(A U C=0.802)$, papillary renal cell $(A \cup C=0.812)$, liver $(A \cup C=0.720)$ and stomach (AUC: 0.728) (Fig. 3C). In terms of predicting prognosis in glioma subtypes, performance of the signature was the best in oligoastrocytoma (AUC $=0.823$ ), followed by glioblastoma multiforme. (AUC $=0.761$ ) and astrocytoma (AUC $=0.743$ ) (Fig. 3c). The signature also performed well when all glioma subtypes were considered as a group ( $A U C=0.815)$ (Fig. 3C).

The Notch-Hedgehog signature identifies molecular subtypes with stem cell-like features

Notch-Hedgehog hyperactivation is associated with increased mortality rates (Figs. 2 and 3). To further investigate the underlying biological consequences of augmented Notch-Hedgehog signalling and how they lead to adverse outcomes, we performed differential expression analyses on all transcripts comparing highand low-risk patients as predicted by the 13-gene signature. The liver cancer cohort had the highest number of differentially expressed genes (DEGs): 3,015 genes $\left(-1.5>\log _{2}\right.$ fold change $>1.5 ; P<0.01)$ (Table S4). This was followed by glioma $(1,407$ genes), stomach (906 genes), papillary renal cell (817 genes) and clear cell renal cell (545 genes) carcinoma (Table S4). Despite having very different pathologies, there was a great deal of DEG overlap between these cancers. 14 DEGs were found in all five cancers, 164 DEGs were observed in at least four cancers and 470 DEGs in at least three cancers (Fig. S3a), implying conserved biological roles of Notch-Hedgehog signalling in driving disease progression.

KEGG pathway analyses on DEGs demonstrated enrichments of pathways involved in regulating self-renewal and pluripotency, i.e. Wnt, TGF- $\beta$, MAPK, JAK-STAT and PPAR signalling (Fig. 3d; Fig. S3b), suggesting that tumours with hyperactive Notch-Hedgehog 


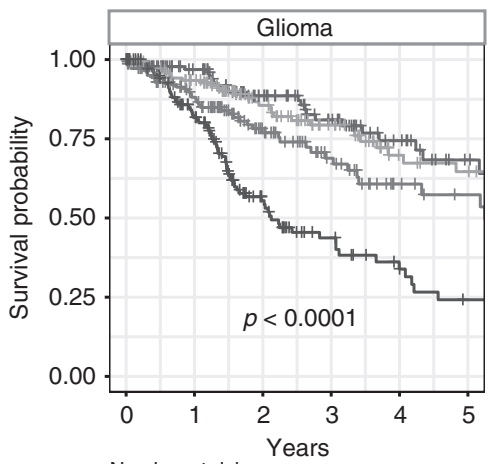

Number at risk

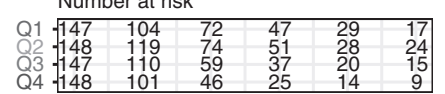

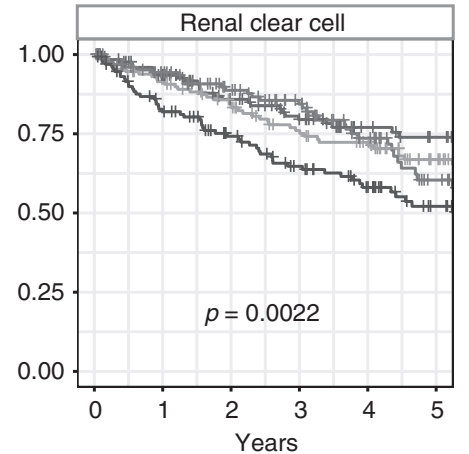

Number at risk

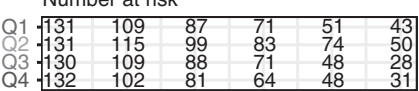

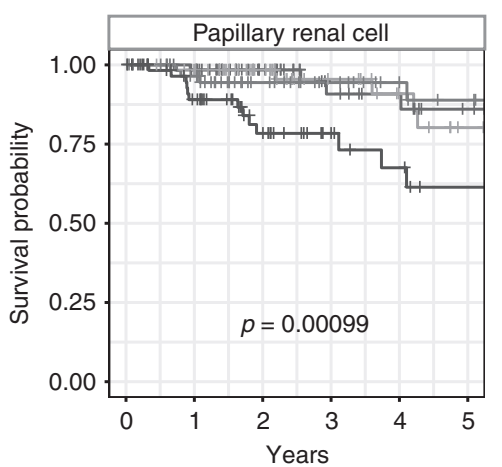

Number at risk

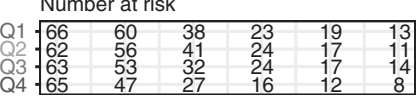

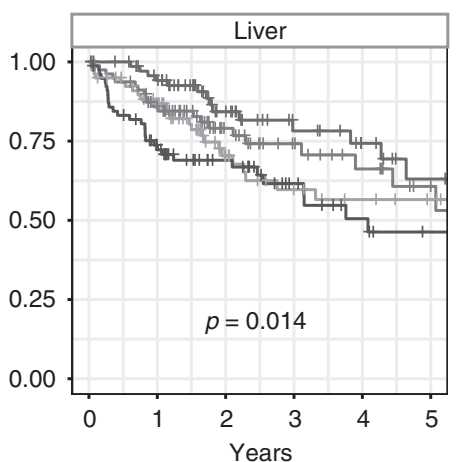

Number at risk

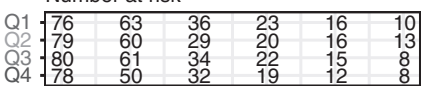

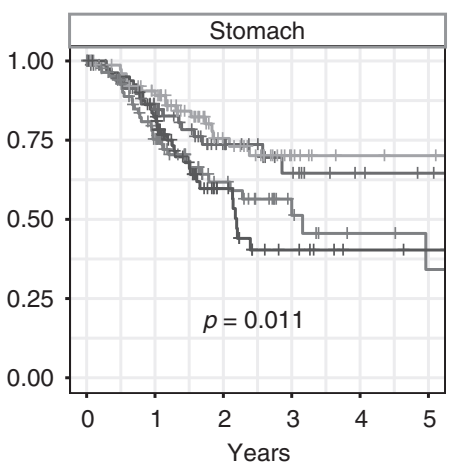

Number at risk

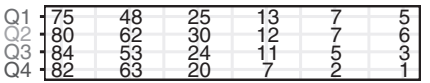

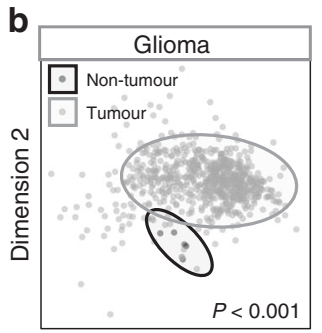
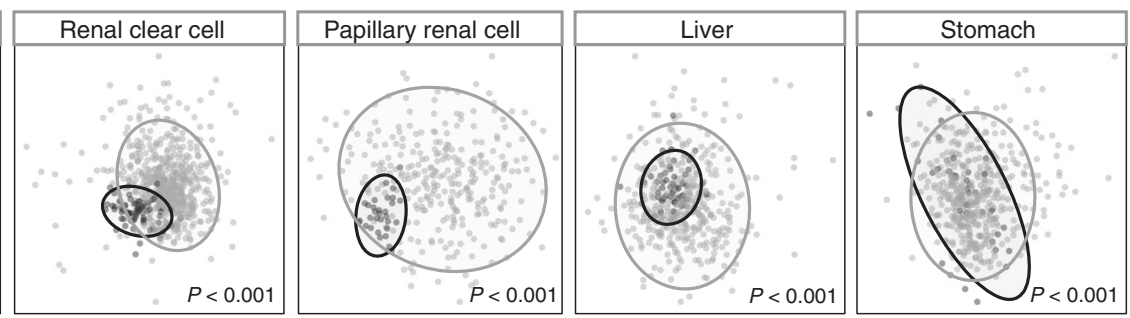

Dimension 1

Fig. 2 The Notch-Hedgehog 13-gene signature predicts patient survival in five cancers. a Kaplan-Meier estimates for overall survival using the signature. Patients were ranked and quartile stratified into Q1 (<25\%), Q2 (25-50\%), Q3 (50-75\%) and Q4 (>75\%) based on their 13-gene scores. $P$-values were determined using the log-rank test. $\mathbf{b}$ Separation of tumour from non-tumour samples using the signature. Ordination plots of MDS analysis of the signature using Euclidean distances to represent tumour and non-tumour samples in 2-dimensional space. PERMANOVA test confirmed statistically significant differences between tumour and non-tumour samples

signalling were characterised by molecular footprints of stemness and that there was significant crosstalk between Notch-Hedgehog and other pathways involved in controlling tumour initiation. ${ }^{28,29}$ Additionally, Gene Ontology analyses revealed significant enrichments of processes related to cell differentiation, cell proliferation, embryo development and morphogenesis (Fig. 3d), supporting the hypothesis that tumour aggression and elevated mortality could be caused by the presence of CSCs that are likely to be refractory to therapy. Consistent with these results, Enrichr transcription factor (TF) analyses revealed that TFs associated with stem cell function appeared amongst top enriched candidates (Fig. 3d). DEGs were enriched as binding targets of SUZ12, REST, EZH2, SMAD4 and FOXM1 as supported by both ChEA and ENCODE databases (Fig. 3d). Binding targets of SUZ12 and $\mathrm{EZH} 2$ were consistently enriched across all five cancer types, while targets of REST and SMAD4 were enriched in all cancers except for clear cell renal cell carcinoma (Fig. 3d). These TFs were thought to induce epithelial-mesenchymal transition and promote invasion and metastasis consistent with their roles in tumour initiation and maintenance. ${ }^{30-32}$

To independently confirm that the 13-gene signature is a potential pan-cancer marker of CSCs, we performed Spearman's correlation analyses to compare 13-gene scores with expression profiles of other CSC markers where we would expect to see positive correlations. We examined expression profiles of nine genes implicated in CSC regulation: CD105, CD133, CD200, CD24, CD29, CD44, CD73, CD90 and NESTIN. Putative neural CSC markers are CD133, NESTIN, CD105 and CD44. ${ }^{33}$ We observed significant positive correlations between 13-gene scores and all four markers in glioma samples (Fig. S4). CD105, CD29, CD44, CD73, CD90 and 

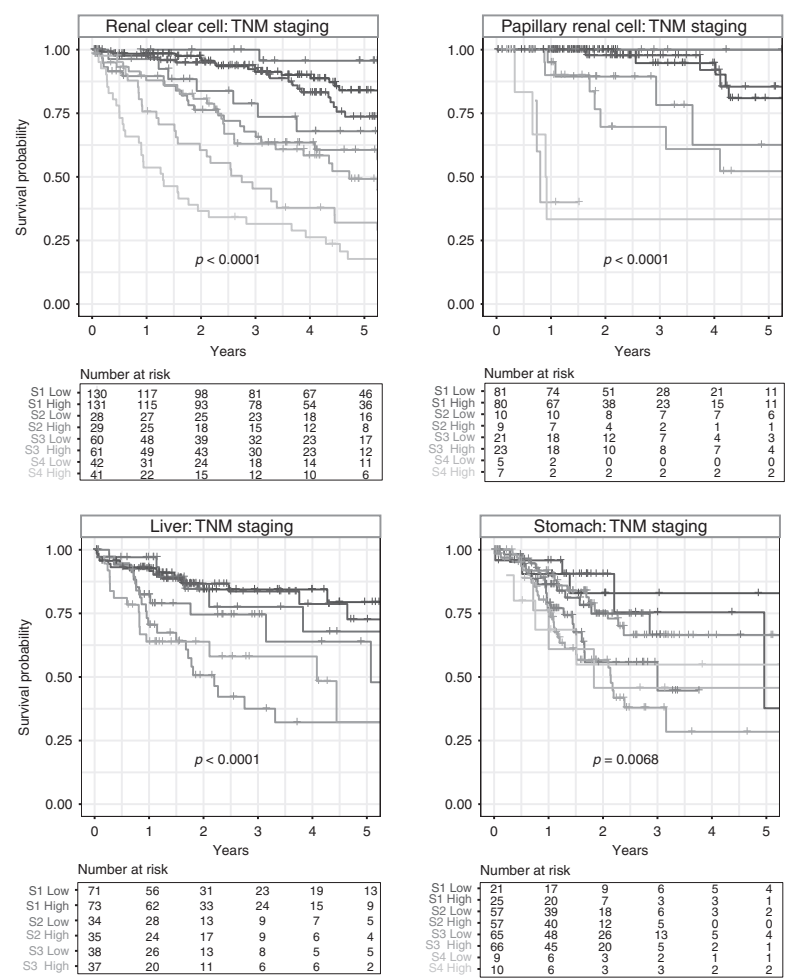

b
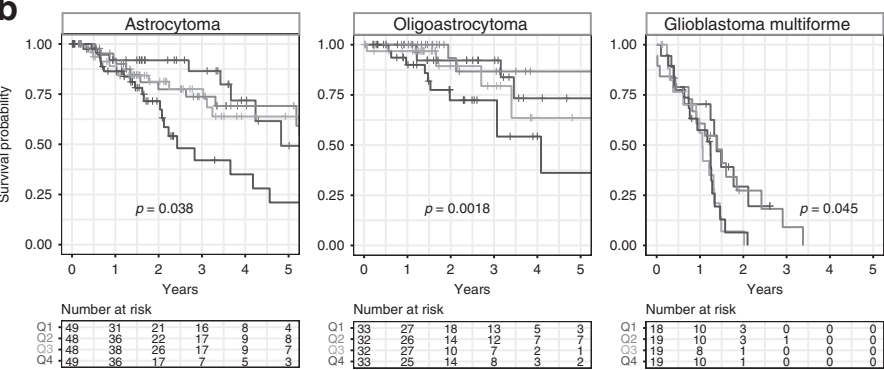

Number at risk

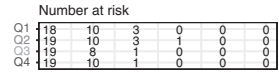

d
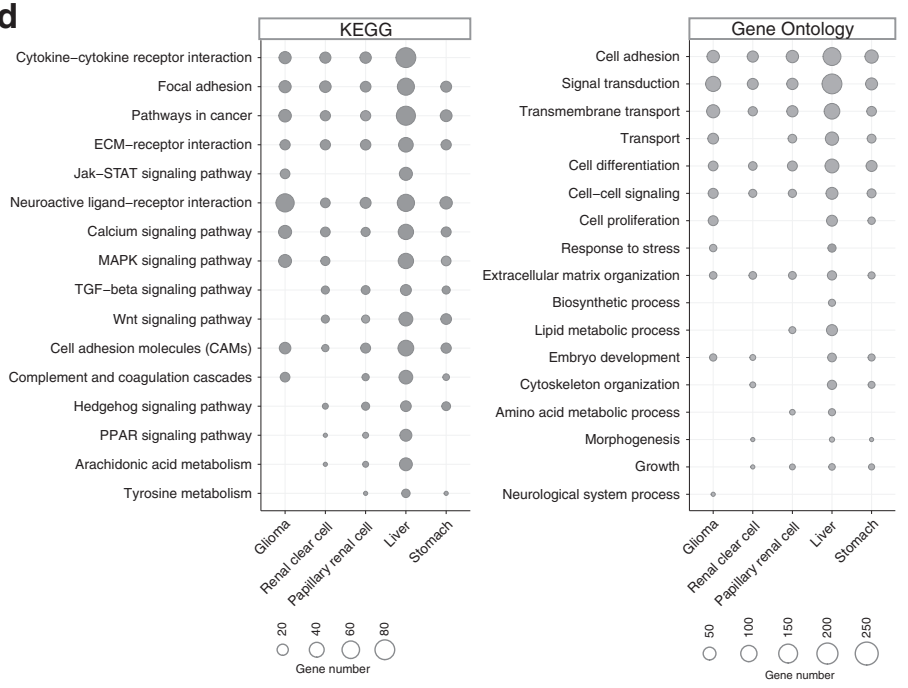

C
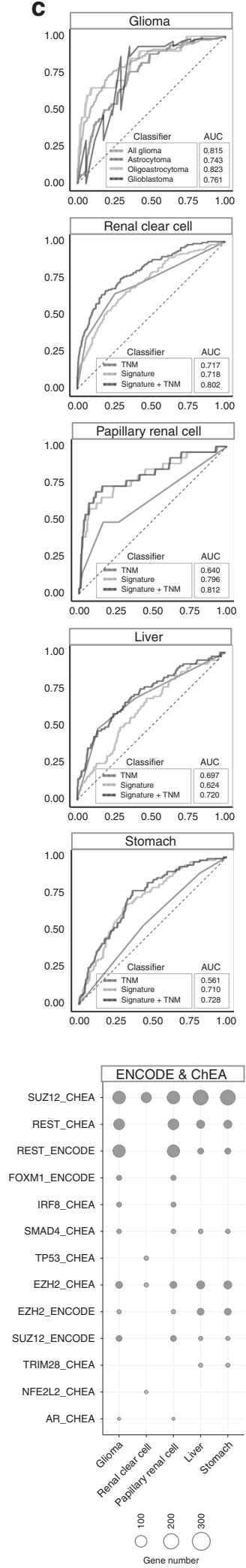

NESTIN were positively correlated with 13-gene scores in renal cancers (Fig. S4); an observation which is consistent with these genes being markers of renal CSCs. ${ }^{34}$ Seven and four CSC markers were positively correlated with 13-gene scores in liver and stomach cancers respectively (Fig. S4). Given the tissue-specific 
Fig. 3 The Notch-Hedgehog 13-gene signature is independent of TNM stage and predicts overall survival in glioma histological subtypes. a Kaplan-Meier analyses were performed on patients stratified according to TNM stages and 13-gene scores. Patients were first separated into TNM stage and then median-stratified into low- and high-score groups based on their 13-gene scores. $P$-values were determined using the log-rank test. b Kaplan-Meier estimates for overall survival using the signature on glioma subtypes ranging from low-grade (astrocytoma, oligoastrocytoma) to high-grade gliomas (glioblastoma multiforme). Patients were first stratified by histological subtypes followed by quartile stratification into Q1 (<25\%), Q2 (25-50\%), Q3 (50-75\%) and Q4 (>75\%) based on their 13-gene scores. P-values were determined using the log-rank test. c Predictive performance of the signature. The receiver operating characteristic (ROC) analysis was used to assess specificity and sensitivity of the signature in predicting 5-year overall survival. ROC curves generated from the signature were compared to those generated from TNM staging and a combined model uniting TNM stage and the signature. AUCs for TNM stage were in accordance with previous publications employing TCGA datasets. ${ }^{2,22,51}$ AUC: area under the curve. TNM: tumour, node and metastasis. $\mathbf{d}$ Enriched biological pathways and transcription factor binding associated with DEGs. Differential expression analyses were performed between Q4 and Q1 patients followed by mapping of DEGs against KEGG, Gene Ontology, ChEA and ENCODE databases

Transcription factors involved in self-renewal processes influence survival outcomes in patients with hyperactive Notch-Hedgehog signalling

Previously, we observed that binding targets of TFs associated with stem cell function were enriched amongst DEGs (Fig. 3d). Polycomb proteins, EZH2 and SUZ12 have been implicated in CSC formation and maintenance. ${ }^{35,36}$ REST is a transcriptional repressor involved in maintaining embryonic and neural stem cell phenotypes. ${ }^{37}$ Given their roles in CSC maintenance, we would expect to see the elevated expression of these TFs in tumours with hyperactive Notch-Hedgehog signalling. Indeed, we observed significant positive correlations between 13-gene scores and EZH2 levels in glioma (rho $=0.45 ; P<0.0001)$, clear cell renal cell ( $r$ ho $=$ 0.22 ; $P<0.0001$ ), papillary renal cell (rho $=0.33 ; P<0.0001)$ and liver (rho $=0.26 ; P<0.0001$ ) cancers (Fig. 4a). Additionally, in the glioma cohort, positive associations between 13-gene scores and REST (rho $=0.39 ; P<0.0001$ ) or SUZ12 (rho $=0.17 ; P<0.0001$ ) profiles were observed (Fig. $4 d$ ).

To determine whether these associations harboured prognostic information, patients were categorised by their 13-gene scores and expression profiles of individual TFs into four categories: (1) high 13-gene score and high TF expression, (2) high 13-gene score and low TF expression, (3) low 13-gene score and high TF expression and (4) low 13-gene score and low TF expression (Fig. 4a, d). Interestingly, combined relationship of the signature and TF expression profiles allowed further delineation of patients into additional risk groups: glioma (EZH2: $P<0.0001 ;$ REST: $P<0.0001$ and SUZ12: $P<0.0001)$, clear cell renal cell (EZH2: $P<0.0001)$, papillary renal cell $(E Z H 2: P=$ $0.029)$ and liver $(E Z H 2: P<0.00057)$ cancers (Fig. $4 \mathrm{~b}$, e). Patients with high 13-gene scores that concurrently harboured high TF expression had the poorest survival outcomes: glioma (EZH2: $\mathrm{HR}$ $=5.141, P<0.0001 ;$ REST: $\mathrm{HR}=3.646, P<0.0001$; SUZ12: $\mathrm{HR}=$ 3.596, $P<0.0001)$, clear cell renal cell $(E Z H 2: H R=2.854, P<$ $0.0001)$, papillary renal cell $(E Z H 2: H R=4.391, P=0.0099)$ and liver $(E Z H 2$ : $H R=2.685, P=0.0005)$ cancers (Fig. 4c, f). Taken together, our results suggest that coregulation by NotchHedgehog signalling and CSC TFs could synergistically contribute to more advanced disease states.

\section{Tumour hypoxia exacerbates disease phenotypes in Notch-} Hedgehog $^{+}$CSCs

Hypoxia is intricately linked to pluripotency as it promotes stem cell maintenance and self-renewal in both embryonic stem cells and $\mathrm{CSCs}^{38}$ in part, through modulating hypoxia-inducible factor (HIF) function. ${ }^{39}$ For example, glioma stem cells are typically found in the vicinity of necrotic regions that are hypoxic. ${ }^{40}$ Glioma stem cells have increased ability to stimulate angiogenesis through VEGF upregulation ${ }^{41}$ and inhibition of HIFs could reduce CSC survival, self-renewal and proliferation. ${ }^{40}$ We reason that hypoxia functions to maintain CSC niches. To assess the levels of tumour hypoxia, we employed a 52-hypoxia gene signature $^{17}$ for calculating hypoxia scores in each patient by taking the average expression of hypoxia signature genes. ${ }^{17}$ Indeed, we observed significant positive correlations between Notch-Hedgehog ${ }^{+}$CSCs and hypoxia scores in glioma (rho $=0.33, P<0.0001$ ) and clear cell renal cell carcinoma (rho $=0.16, P=0.00031$ ) (Fig. 5a). By grouping patients based on their 13-gene and hypoxia scores, this joint model allowed the identification of patients with potentially more hypoxic tumours harbouring Notch-Hedgehog ${ }^{+}$CSCs, which influenced overall survival rates: glioma $(P<0.0001)$ and clear cell renal cell carcinoma ( $P=0.00013)$ (Fig. 5b). Indeed, patients with high CSC and hypoxia scores had significantly poorer survival outcomes: glioma $(\mathrm{HR}=6.008 ; P<0.0001)$ and clear cell renal cell carcinoma ( $\mathrm{HR}=2.389, P<0.0001)$ (Fig. 5c). The CSC-hypoxia model is also prognostic in glioma subtypes: astrocytoma ( $\mathrm{HR}=$ $5.052, P<0.0001)$, oligoastrocytoma $(\mathrm{HR}=16.717, P=0.0066)$ and glioblastoma $(\mathrm{HR}=2.686, P=0.022) \quad$ (Fig. 5b, c). Our results suggest that hypoxic zones within tumours could very well represent CSC niches.

\section{Putative Notch-Hedgehog ${ }^{+}$CSCs are potentially immune} privileged

Cancer progression is negatively correlated with immunocompetence of the host and evidence points to the role of CSCs in immunomodulation. ${ }^{3,42}$ CSCs reside within niches that are often protected from environmental insults as well as attacks by the immune system. Hypoxic zones not only serve as CSC niches (Fig. 5), ${ }^{43}$ but also attract immunosuppressive cells such as regulatory T cells (Tregs), ${ }^{22,44}$ tumour-associated macrophages ${ }^{45}$ and myeloid-derived suppressor cells. ${ }^{46}$ Given that positive associations between Notch-Hedgehog ${ }^{+}$CSCs and hypoxia were linked to poor progression in glioma and clear cell renal cell carcinoma, we hypothesise that tumours characterised by these features would be immune privileged or hypoimmunogenic.

To test this hypothesis, we retrieved a list of 31 genes that represent tumour-infiltrating Tregs. This gene list was identified from the overlap of four Treg signatures ${ }^{18-21}$ to yield a more representative profile of tumour-infiltrating Tregs that is not specific to a single cancer type. A Treg score for each patient within the glioma and clear cell renal cell carcinoma cohorts was calculated as the mean expression of the 31 genes. We observed significant positive correlations between Treg scores and the Notch-Hedgehog 13-gene scores in both cohorts, supporting the hypothesis that CSCs are potentially hypoimmunogenic: glioma (rho $=0.43 ; P<0.0001$ ) and clear cell renal cell carcinoma (rho $=$ $0.31 ; P<0.0001$ ) (Fig. 6a). As performed previously, patients were separated into four groups based on their 13-gene and Treg scores. When used in combination with the Notch-Hedgehog signature, Treg expression profiles allowed further separation of patients into additional risk groups that influenced overall survival: glioma $(P<0.0001)$ and clear cell renal cell carcinoma $(P<0.0001)$ (Fig. 6b). Intriguingly, patients characterised by high 13-gene and Treg scores had significantly higher mortality rates compared to those with low 13-gene and Treg scores: glioma $(H R=4.921, P<$ $0.0001)$ and clear cell renal cell carcinoma $(H R=2.968, P<0.0001)$ (Fig. 6c). This was also true for other histological subtypes of glioma: astrocytoma $(\mathrm{HR}=2.721 ; P=0.0032)$, oligoastrocytoma 
a

High signature score and low EZH2 expression

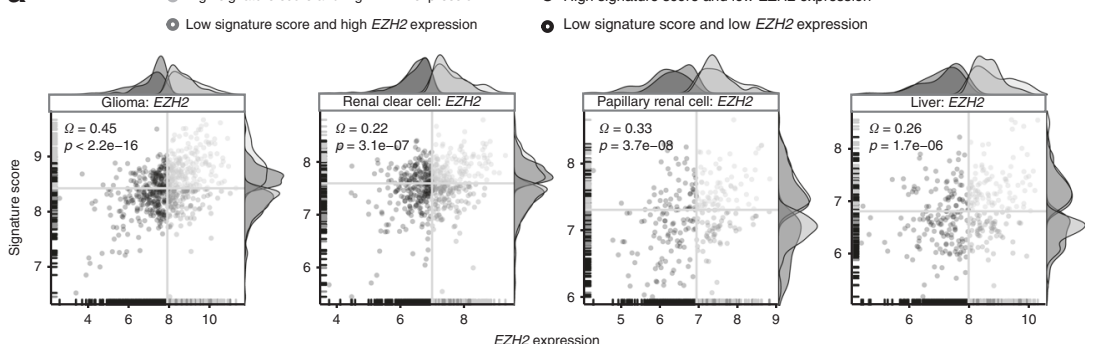

b - High signature score and high EZH2 expression - High signature score and low EZH2 expression
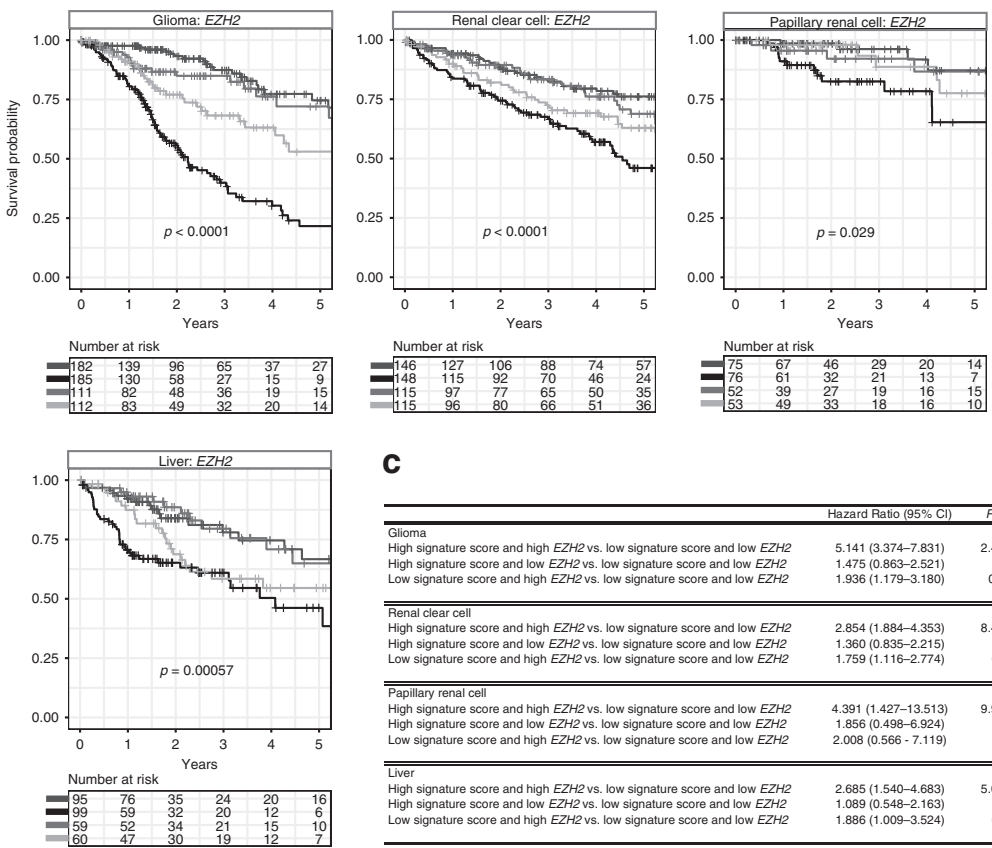

C

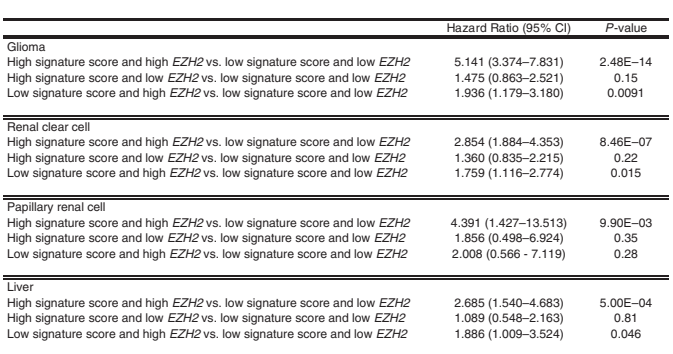

d
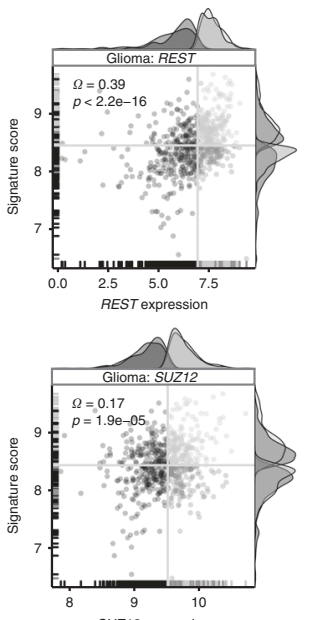

High signature score and high REST/SUZ12 expression - Low signature score and high REST/SUZ12 expression - High signature score and low REST/SUZ12 expression - Low signature score and low REST/SUZ12 expression e

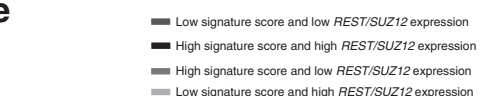

Low signature score and high REST/SUZ12 expression
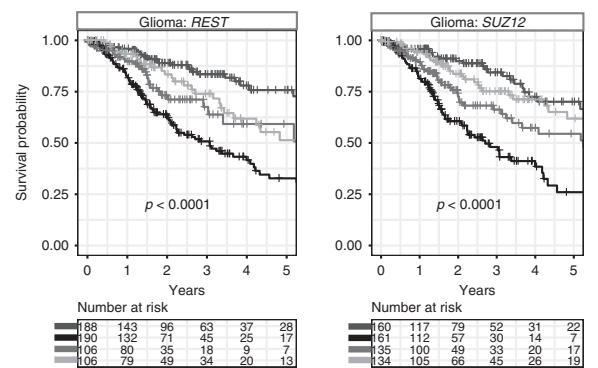

f

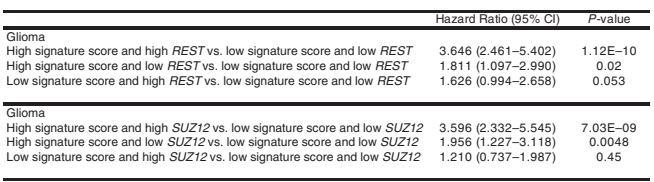

$(\mathrm{HR}=5.431 ; P=0.0091)$ and glioblastoma $(\mathrm{HR}=3.065 ; \quad P=$ 0.0068) (Fig. 6c). Taken together, our results suggest that CSCs found within immunosuppressed environments are likely to be more aggressive.

\section{DISCUSSION AND CONCLUSION}

Aberrations in the Notch-Hedgehog signalling axis are frequently implicated in malignant progression. Hedgehog genes, Shh, PTCH1 and GLI1, were detected in over 50\% of liver cancer tumours and 
674

Fig. 4 Prognostic significance of a combined model of the Notch-Hedgehog signature and transcription factors (EZH2, SUZ12 and REST) involved in pluripotency maintenance. a Scatter plots demonstrate significant positive correlations between 13-gene scores and EZH2 expression profiles in four cancers. Patients were stratified into four categories based on median 13-gene scores and EZH2 expression. Density plots depict the distribution of 13-gene scores and EZH2 expression at the $y$ - and $x$-axes, respectively. b Kaplan-Meier analyses were performed on the four patient categories to ascertain the combined relationship of the signature and EZH2 expression on overall survival. c Table inset depicts univariate Cox proportional hazards analyses of the relationship between EZH2 and the signature in four cancer types. d Scatter plots demonstrate significant positive correlations between 13-gene scores and REST or SUZ12 expression levels in glioma. Patients were stratified into four categories based on median 13-gene score and REST or SUZ12 expression. Density plots depict the distribution of 13gene scores and REST or SUZ12 expression at the $y$ - and $x$-axes, respectively. e Kaplan-Meier analyses were performed on the four patient categories to ascertain the combined relationship between the signature and REST or SUZ12 expression on overall survival in glioma. $f$ Table inset depicts univariate Cox proportional hazards analyses of the relationship between REST or SUZ12 and the signature in glioma. Cl: confidence interval. Significant $P$-values are highlighted in bold

inhibition of Hedgehog signalling by cyclopamine, Smoothened antagonist or anti-SHH resulted in decreased cell growth and increased apoptosis. ${ }^{47}$ Notch signalling is also activated in liver cancer and this leads to the formation of liver tumours in mice. ${ }^{48}$ Notch blockade using $\mathrm{Y}$-secretase inhibitors reduced cell viability in hepatoma cell lines. ${ }^{48}$ In clear cell renal cell carcinoma, inhibition of Notch signalling reduced anchorage-independent growth and mice treated with Notch inhibitors had impaired growth of transplanted cancer cells. ${ }^{49}$ Elevated expression of Notch ligands correlated with aggressiveness and poor survival rates in stomach cancer. ${ }^{50}$

These studies have paved the road for understanding the role of Notch-Hedgehog signalling in carcinogenesis. However, largescale comparative studies investigating the similarities and differences in Notch-Hedgehog signalling across multiple cancer types have remained limited. We interrogated expression and mutational profiles of 72 genes from Notch and Hedgehog pathways in 21 diverse cancer types involving 18,484 patients. Our integrated analysis of genomic, transcriptomic and clinical data revealed molecular distinct tumour subtypes that were characterised by Notch-Hedgehog hyperactivation. Concentrating on 13 Notch-Hedgehog driver genes that were recurrently amplified and overexpressed, we found that these genes were associated with clinically relevant molecular features of stemness. The biological consequences of elevated expression of driver genes were manifold. High-risk patients showed overexpression of genes associated with other stem cell-related pathways such as Wnt, JAK-STAT and TGF- $\beta$ signalling (Fig. $3 \mathrm{~d}$. and S3b). ${ }^{51}$ Simultaneous inhibition of Notch and JAK-STAT pathways by combined AG-490 and GSI IX therapy impaired pancreatic cancer progression. ${ }^{52}$ GLI2 is regulated by both Hedgehog and TGF- $\beta$ pathways and others have surmised that TGF- $\beta$ may potentiate Hedgehog signalling cascade by increasing GL12 availability, contributing to metastasis. ${ }^{53}$ Hence, our study reveals molecular targets with overlapping functions that can be prioritised to improve therapeutic outcomes.

Furthermore, binding targets of stem cell-related TFs $(E Z H 2$, SUZ12 and REST) were enriched amongst genes upregulated in high-risk patients (Fig. 4). EZH2 synergises with Notch-Hedgehog ${ }^{+}$ CSCs to worsen survival outcomes in patients with glioma, renal and liver cancers (Fig. 4). Pharmacological inhibition of EZH2 impaired glioblastoma CSC tumour-initiating capacity and survival. $^{35}$ EZH2-mediated transcriptional silencing leads to the maintenance of undifferentiated states in glioblastoma through STAT3 activation. ${ }^{54}$ In liver cancer, EZH2 overexpression is associated with vascular invasion, malignant progression ${ }^{55}$ and activation of $\beta$-catenin/Wnt signalling. ${ }^{56}$ Inhibition of $E Z H 2$ in renal cancer cell lines led to increased apoptosis. ${ }^{57}$ Additionally, enrichments of SUZ12 and REST targets in glioma patients with hyperactive Notch-Hedgehog signalling were linked to significantly poorer prognosis (Fig. 4d, e). REST is implicated in transcriptional regulation of neuronal stem cells, ${ }^{37}$ while the overexpression of SUZ12 is linked to tumour progression. ${ }^{58}$

An exploration of the relationship between Notch-Hedgehog hyperactivation and tumour microenvironmental qualities revealed associations of CSCs with hypoxia and immunosuppression. We observed that CSCs characterised by hyperactive NotchHedgehog signalling exhibited immune privileged features associated with the attenuation effects of Tregs (Fig. 6). Effectiveness of immunotherapy is biased towards differentiated cells that make up the tumour bulk due to distinct antigen presentation in $\mathrm{CSCs}^{59} \mathrm{CD}^{533^{+}}$glioma CSCs fail to express NK cell ligands or $\mathrm{MHCl}$, which prevents immune detection. ${ }^{60}$ Stimulatory NK cell ligands are also downregulated in breast CSCs, contributing to evasion from NK cell killing. ${ }^{61}$ Pan et al. elegantly reviewed recent initiatives focusing on immunotherapeutic agents against CSC antigens employing dendritic cell vaccines, myeloid-derived suppressor cell-based approaches and the use of immune checkpoint blockades recognising $P D-1$ or CTLA4. ${ }^{59}$ The NotchHedgehog signature may be used to stratify patients prior to immunotherapy.

Immunoevasion can be exacerbated by tumour hypoxia as the latter not only promotes CSC survival, but also creates an environment that facilitates further immune suppression. ${ }^{22}$ It may be possible that Notch-Hedgehog ${ }^{+}$CSCs in glioma and clear cell renal cell carcinomas are more frequently found within immunosuppressed hypoxic zones (Fig. 5). Indeed, hypoxia could stimulate self-renewal of $\mathrm{CD}_{133^{+}}$glioma stem cells and this is abrogated by HIF-1a knockdown. ${ }^{62}$ Hypoxia promotes the maintenance of undifferentiated states through the activation of Notch-responsive genes in neuronal progenitors. ${ }^{39}$ Hypoxia also activates cellular reprogramming of non-stem cancer cells into CSCs in glioblastoma by inducing the expression of Oct4, Nanog and $c-M y c{ }^{63}$ Glioma stem cells are pro-angiogenic due to promiscuous secretions of VEGF that is further induced by hypoxia. ${ }^{41}$ Bevacizumab, which targets VEGF, could suppress xenographs derived from glioma stem cells but not those derived from non-stem glioma cells. ${ }^{41}$ In renal cancer, we observed that Notch-Hedgehog ${ }^{+}$CSCs are likely to be enriched in hypoxic tumours and the combined effects of hypoxia and augmented Notch-Hedgehog signalling resulted in further elevation of death risks (Fig. 5). However, Sjölund et al. observed that Notch signalling is not enhanced by hypoxia in renal cancer. ${ }^{49}$ Another study on renal CSCs revealed that hypoxia did not affect the differentiation potential of $\mathrm{CD}_{105}{ }^{+} \mathrm{CSCs}^{64}$ Nonetheless, hypoxia was found to induce the expression of stem cell markers, Oct4, Nanog, c-Myc and KIf4 in renal cancer cell lines, supporting our observation, and in another ten cancers including cervix, lung, colon, liver and prostate. ${ }^{65}$

Although prospective validation is warranted, the results presented in this work support a model where Notch-Hedgehog hyperactivation is linked to stemness and that hypoxia contributes to the maintenance of undifferentiated phenotypes and the reduction of anti-tumour immunity. The use of immune checkpoint blockade has been increasingly tried in malignancy. ${ }^{66}$ Hence, molecular signatures capable of discerning responders from non-responders will be valuable prior to the administration of these expensive drugs. As an independent prognostic indicator in five cancer types involving 2278 patients, the Notch-Hedgehog 

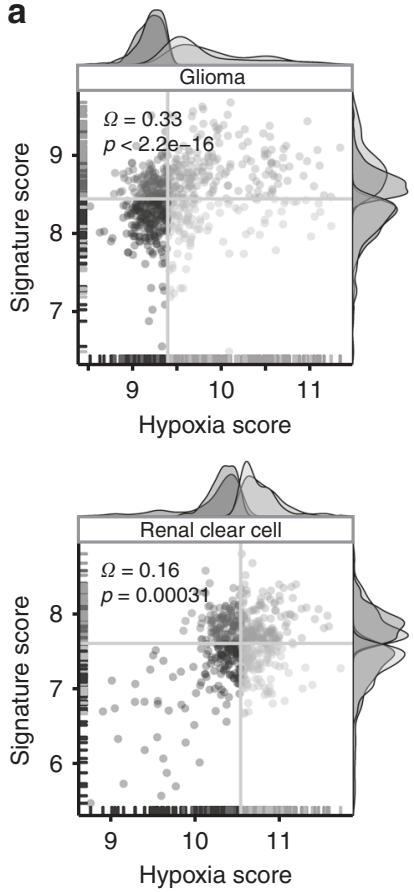

- High signature and high hypoxia scores

Low signature and high hypoxia scores
- High signature and low hypoxia scores

- Low signature and low hypoxia scores

C

\begin{tabular}{|c|c|c|}
\hline & Hazard Ratio $(95 \% \mathrm{Cl})$ & $P$-value \\
\hline \multicolumn{3}{|l|}{ Glioma } \\
\hline High signature and high hypoxia scores vs. low signature and low hypoxia scores & $6.008(3.939-9.161)$ & $2.00 \mathrm{E}-16$ \\
\hline High signature and low hypoxia scores vs. low signature and low hypoxia scores & $0.996(0.545-1.821)$ & 0.98 \\
\hline Low signature and high hypoxia scores vs. low signature and low hypoxia scores & $2.048(1.245-3.367)$ & 0.0047 \\
\hline \multicolumn{3}{|l|}{ Astrocytoma } \\
\hline High signature and high hypoxia scores vs. low signature and low hypoxia scores & $5.052(2.292-11.134)$ & $5.89 \mathrm{E}-05$ \\
\hline High signature and low hypoxia scores vs. low signature and low hypoxia scores & $1.578(0.589-4.223)$ & 0.36 \\
\hline Low signature and high hypoxia scores vs. low signature and low hypoxia scores & $3.827(1.571-9.322)$ & 0.0031 \\
\hline \multicolumn{3}{|l|}{ Oligoastrocytoma } \\
\hline High signature and high hypoxia scores vs. low signature and low hypoxia scores & $16.717(2.189-127.670)$ & $6.60 \mathrm{E}-03$ \\
\hline High signature and low hypoxia scores vs. low signature and low hypoxia scores & $4.310(0.447-41.540)$ & 0.21 \\
\hline Low signature and high hypoxia scores vs. low signature and low hypoxia scores & $5.383(0.646-44.830)$ & 0.12 \\
\hline \multicolumn{3}{|l|}{ Glioblastoma } \\
\hline High signature and high hypoxia scores vs. low signature and low hypoxia scores & $2.686(1.151-6.270)$ & $2.20 \mathrm{E}-02$ \\
\hline High signature and low hypoxia scores vs. low signature and low hypoxia scores & $1.635(0.771-3.473)$ & 0.2 \\
\hline Low signature and high hypoxia scores vs. low signature and low hypoxia scores & $0.799(0.363-1.759)$ & 0.58 \\
\hline \multicolumn{3}{|l|}{ Renal clear cell } \\
\hline High signature and high hypoxia scores vs. low signature and low hypoxia scores & $2.389(1.575-3.625)$ & 4.23E-05 \\
\hline High signature and low hypoxia scores vs. low signature and low hypoxia scores & $1.330(0.829-2.135)$ & 0.24 \\
\hline Low signature and high hypoxia scores vs. low signature and low hypoxia scores & $1.350(0.857-2.126)$ & 0.19 \\
\hline
\end{tabular}

b
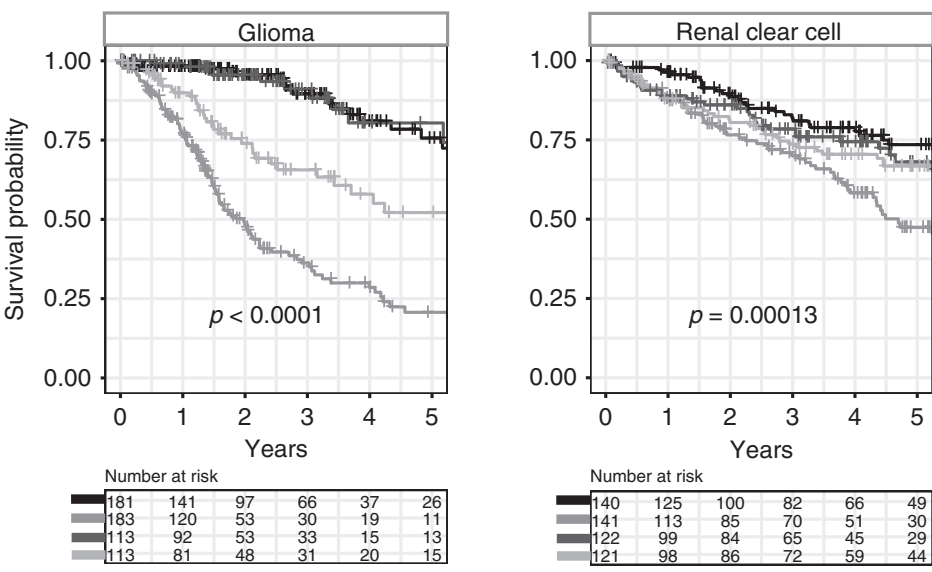

Low signature and low hypoxia scores

High signature and high hypoxia scores

- High signature and low hypoxia scores

Low signature and high hypoxia scores
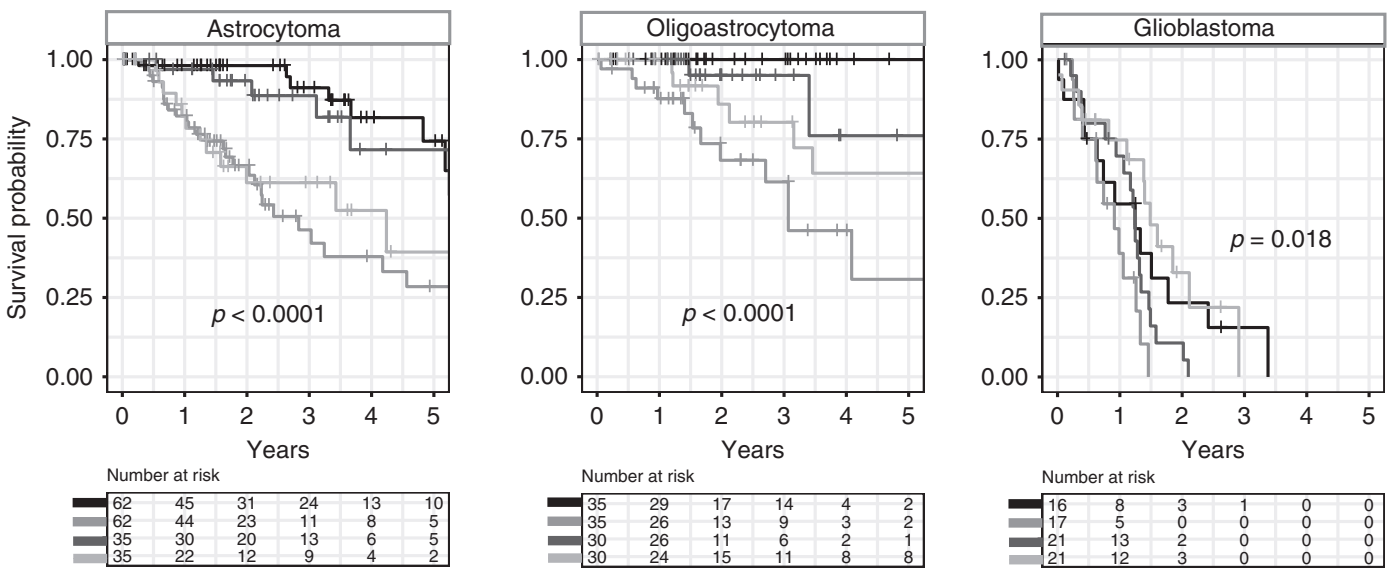

Fig. 5 Positive associations between Notch-Hedgehog ${ }^{+} \operatorname{CSCs}$ and tumour hypoxia in glioma and clear cell renal cell carcinoma. a Scatter plots demonstrate significant positive correlations between 13-gene and hypoxia scores. Patients were stratified into four categories based on median 13gene and hypoxia scores. Density plots depict the distribution of 13-gene and hypoxia scores at the $y$ - and $x$-axes, respectively. $\mathbf{b}$ Kaplan-Meier analyses were performed on the four patient categories to ascertain the combined relationship of the signature and tumour hypoxia on overall survival. Contribution of hypoxia on Notch-Hedgehog ${ }^{+}$CSCs were also determined in histological subtypes of glioma (astrocytoma, oligoastrocytoma and glioblastoma multiforme). c Table inset demonstrates univariate Cox proportional hazards analyses of the relationship between tumour hypoxia and the signature in glioma and clear cell renal cell carcinoma. Cl: confidence interval. Significant $P$-values are highlighted in bold 


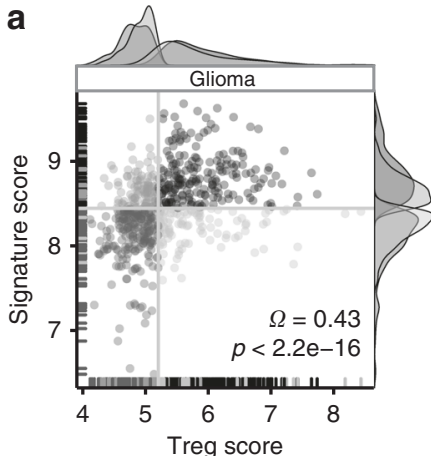

- High signature and high Treg scores

- Low signature and high Treg scores
- High signature and low Treg scores

- Low signature and low Treg scores

C

\begin{tabular}{|c|c|c|}
\hline & Hazard Ratio $(95 \% \mathrm{Cl})$ & $P$-value \\
\hline \multicolumn{3}{|l|}{ Glioma } \\
\hline High signature and high Treg scores vs. low signature and low Treg scores & $4.921(3.277-7.391)$ & $1.60 \mathrm{E}-14$ \\
\hline High signature and low Treg scores vs. low signature and low Treg scores & $1.671(0.989-2.822)$ & 0.055 \\
\hline Low signature and high Treg scores vs. low signature and low Treg scores & $2.377(1.459-3.872)$ & 0.000503 \\
\hline \multicolumn{3}{|l|}{ Astrocytoma } \\
\hline High signature and high Treg scores vs. low signature and low Treg scores & $2.721(1.398-5.298)$ & $3.20 \mathrm{E}-03$ \\
\hline High signature and low Treg scores vs. low signature and low Treg scores & $1.288(0.561-2.960)$ & 0.55 \\
\hline Low signature and high Treg scores vs. low signature and low Treg scores & $1.457(0.597-3.554)$ & 0.41 \\
\hline \multicolumn{3}{|l|}{ Oligoastrocytoma } \\
\hline High signature and high Treg scores vs. low signature and low Treg scores & $5.431(1.522-19.374)$ & $9.10 \mathrm{E}-03$ \\
\hline High signature and low Treg scores vs. low signature and low Treg scores & $2.470(0.576-10.603)$ & 0.22 \\
\hline Low signature and high Treg scores vs. low signature and low Treg scores & $1.032(0.222-4.804)$ & 0.97 \\
\hline \multicolumn{3}{|l|}{ Glioblastoma } \\
\hline High signature and high Treg scores vs. low signature and low Treg scores & $3.065(1.362-6.900)$ & 0.0068 \\
\hline High signature and low Treg scores vs. low signature and low Treg scores & $1.955(0.824-4.639)$ & 0.13 \\
\hline Low signature and high Treg scores vs. low signature and low Treg scores & $1.274(0.570-2.846)$ & 0.55 \\
\hline \multicolumn{3}{|l|}{ Renal clear cell } \\
\hline High signature and high Treg scores vs. low signature and low Treg scores & $2.968(1.922-4.582)$ & $9.20 \mathrm{E}-07$ \\
\hline High signature and low Treg scores vs. low signature and low Treg scores & $1.649(0.997-2.728)$ & 0.051 \\
\hline Low signature and high Treg scores vs. low signature and low Treg scores & $2.132(1.342-3.385)$ & 0.0013 \\
\hline
\end{tabular}

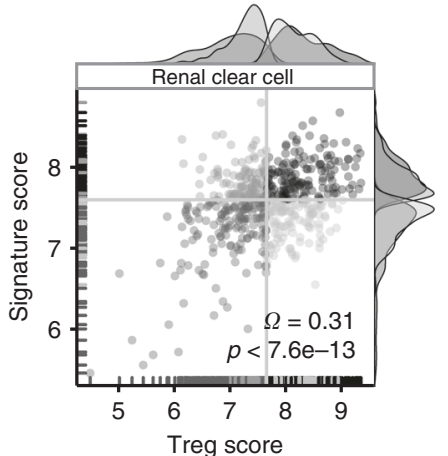
High signature and low Treg scores vs. low signature and low Treg scores

$9.10 \mathrm{E}-03$ 0.22

Glioblastoma

$3.065(1.362-6.900) \quad 0.0068$ $.955(0.824-4.639)-0.13$

$9.968(1.922-4.582) \quad 9.20 \mathrm{E}-07$ $649(0.997-2.728)$ 0.051 High signature and low Treg scores vs. low signature and low Treg scores

b

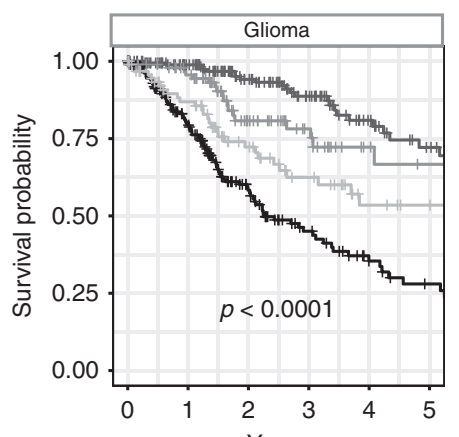

Number at risk
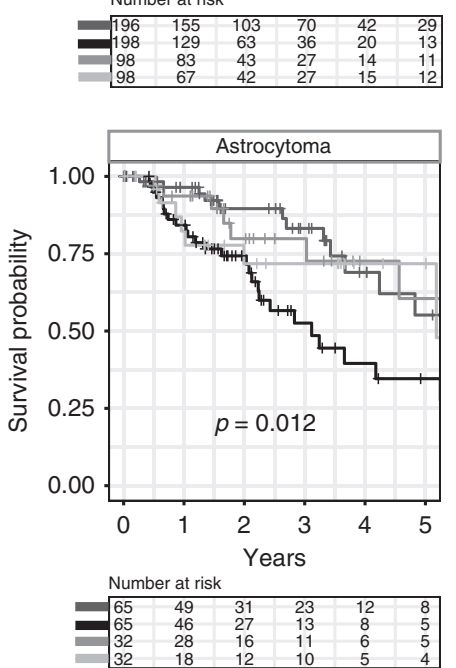

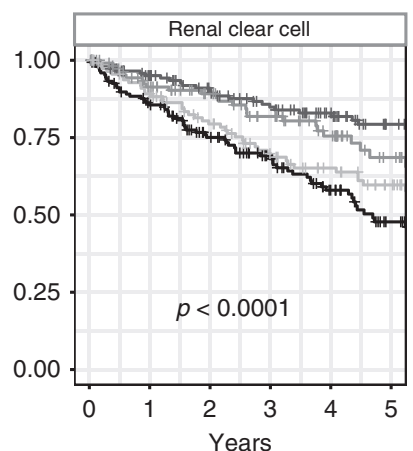

Low signature and low Treg scores

- High signature and high Treg scores

- High signature and low Treg scores

Low signature and high Treg scores
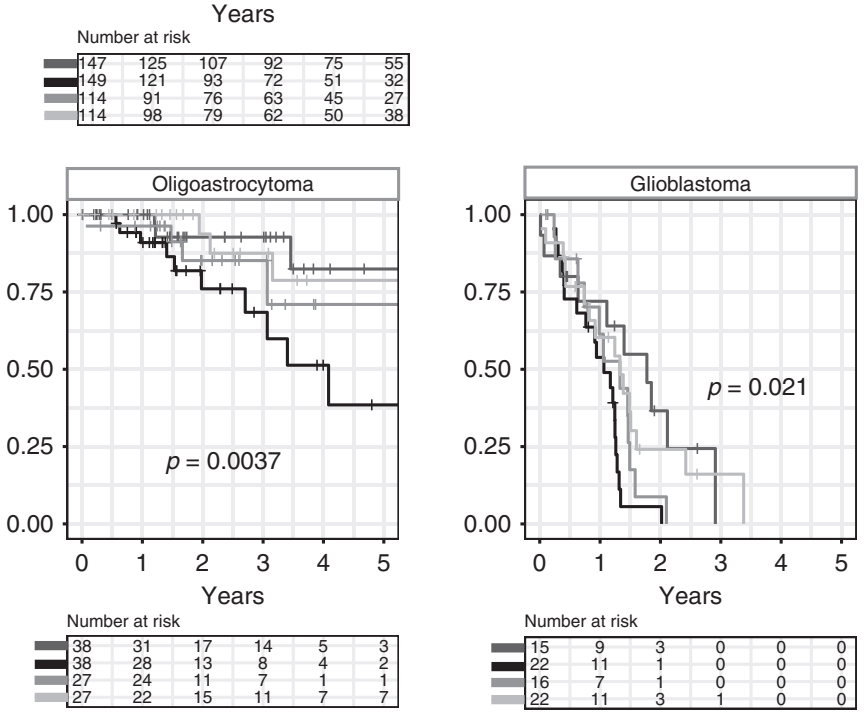
Fig. 6 Positive associations between Notch-Hedgehog ${ }^{+}$CSCs and immunosuppression in glioma and clear cell renal cell carcinoma. a Scatter plots demonstrate significant positive correlations between 13-gene and Treg scores. Patients were stratified into four categories based on median 13-gene and Treg scores. Density plots depict the distribution of 13-gene and Treg scores at the $y$-and $x$-axes respectively. b Kaplan-Meier analyses were performed on the four patient categories to ascertain the combined relationship of the signature and Tregmediated immunosuppression on overall survival. Contribution of Tregs on Notch-Hedgehog ${ }^{+}$CSCs were also determined in histological subtypes of glioma (astrocytoma, oligoastrocytoma and glioblastoma multiforme). c Table inset demonstrates univariate Cox proportional hazards analyses of the relationship between Tregs and the signature in glioma and clear cell renal cell carcinoma. Cl: confidence interval. Significant $P$-values are highlighted in bold

gene signature may serve as a staging point for exploring combinatorial treatments that simultaneously target CSCs, hypoxia and tumour immunity.

\section{AUTHOR CONTRIBUTIONS}

A.G.L. designed the study and supervised the research. W.H.C. and A.G.L. analysed the data, interpreted the results and wrote the initial manuscript draft. A.G.L. revised the manuscript draft. All authors approved the final submitted manuscript.

\section{ADDITIONAL INFORMATION}

Supplementary information is available for this paper at https://doi.org/10.1038/ s41416-019-0572-9.

Competing interests: The authors declare no competing interests.

Ethics approval and consent to participate: No ethics approval or consent to participate is required as this study is conducted using publicly available datasets from The Cancer Genome Atlas.

Funding: None

Consent to publish: None.

Data availability: The datasets supporting the conclusions of this article are included within the article and its Supplementary files.

Note: This work is published under the standard license to publish agreement. After 12 months the work will become freely available and the license terms will switch to a Creative Commons Attribution 4.0 International (CC BY 4.0).

Publisher's note Springer Nature remains neutral with regard to jurisdictional claims in published maps and institutional affiliations.

\section{REFERENCES}

1. Maugeri-Saccà, M., Bartucci, M. \& De Maria, R. DNA damage repair pathways in cancer stem cells. Mol. Cancer Ther. 11, 1627-1636 (2012).

2. Chang, W. H. \& Lai, A. G. Transcriptional landscape of DNA repair genes underpins a pan-cancer prognostic signature associated with cell cycle dysregulation and tumor hypoxia. DNA Repair (Amst) [Internet]. 78(Jun), 142-153 (2019).

3. Schatton, T. \& Frank, M. H. Antitumor immunity and cancer stem cells. Ann. $N$ Y Acad. Sci. 1176, 154-169 (2009).

4. Clevers, $\mathrm{H}$. The cancer stem cell: premises, promises and challenges. Nat. Med. 17, 313 (2011)

5. Takebe, N., Harris, P. J., Warren, R. Q. \& Ivy, S. P. Targeting cancer stem cells by inhibiting Wnt, Notch, and Hedgehog pathways. Nat. Rev. Clin. Oncol. 8, 97 (2011).

6. Ellisen, L. W., Bird, J., West, D. C., Soreng, A. L., Reynolds, T. C., Smith, S. D. et al. TAN-1, the human homolog of the Drosophila notch gene, is broken by chromosomal translocations in T lymphoblastic neoplasms. Cell. 66, 649-661 (1991).

7. Lobry, C., Oh, P. \& Aifantis, I. Oncogenic and tumor suppressor functions of Notch in cancer: it's NOTCH what you think. J. Exp. Med. 208, 1931-1935 (2011).

8. Briscoe, J. \& Thérond, P. P. The mechanisms of Hedgehog signalling and its roles in development and disease. Nat. Rev. Mol. Cell Biol. 14, 416 (2013).

9. Solecki, D. J., Liu, X., Tomoda, T., Fang, Y. \& Hatten, M. E. Activated Notch2 signaling inhibits differentiation of cerebellar granule neuron precursors by maintaining proliferation. Neuron. 31, 557-568 (2001).
10. Dakubo, G. D., Mazerolle, C. J. \& Wallace, V. A. Expression of Notch and Wnt pathway components and activation of Notch signaling in medulloblastomas from heterozygous patched mice. J. Neurooncol. 79, 221-227 (2006).

11. Katoh, M. Networking of WNT, FGF, Notch, BMP, and Hedgehog signaling pathways during carcinogenesis. Stem Cell Rev. 3, 30-38 (2007).

12. Steg, A. D., Katre, A. A., Goodman, B. W., Han, H.-D., Nick, A. M., Stone, R. L. et al Targeting the notch ligand JAGGED1 in both tumor cells and stroma in ovarian cancer. Clin. Cancer Res. 17, 5674-5685 (2011). clincanres-0432.

13. Domingo-Domenech, J., Vidal, S. J., Rodriguez-Bravo, V., Castillo-Martin, M., Quinn, S. A., Rodriquez-Barrueco, R. et al. Suppression of acquired docetaxel resistance in prostate cancer through depletion of notch-and hedgehog-dependent tumorinitiating cells. Cancer Cell. 22, 373-388 (2012).

14. Schreck, K. C., Taylor, P., Marchionni, L., Gopalakrishnan, V., Bar, E. E., Gaiano, N. et al. The Notch target Hes1 directly modulates Gli1 expression and Hedgehog signaling: a potential mechanism of therapeutic resistance. Clin. Cancer Res. 16 6060-6070 (2010).

15. Weinstein, J. N., Collisson, E. A., Mills, G. B., Shaw, K. R. M., Ozenberger, B. A., Ellrott, K. et al. The cancer genome atlas pan-cancer analysis project. Nat. Genet. 45, 1113 (2013).

16. Mermel, C. H., Schumacher, S. E., Hill, B., Meyerson, M. L., Beroukhim, R. \& Getz, G. GISTIC2. 0 facilitates sensitive and confident localization of the targets of focal somatic copy-number alteration in human cancers. Genome Biol. 12, R41 (2011).

17. Buffa, F. M., Harris, A. L., West, C. M. \& Miller, C. J. Large meta-analysis of multiple cancers reveals a common, compact and highly prognostic hypoxia metagene. Br. J. Cancer [Internet]. 102, 428-435 (2010).

18. Zheng, C., Zheng, L., Yoo, J. K., Guo, H., Zhang, Y., Guo, X. et al. Landscape of infiltrating $T$ cells in liver cancer revealed by single-cell sequencing. Cell 169 1342-1356.e16 (2017).

19. De Simone, M., Arrigoni, A., Rossetti, G., Gruarin, P., Ranzani, V., Politano, C. et al. Transcriptional Landscape of Human Tissue Lymphocytes Unveils Uniqueness of Tumor-Infiltrating T Regulatory Cells. Immunity 45, 1135-1147 (2016).

20. Plitas, G., Konopacki, C., Wu, K., Bos, P. D., Morrow, M., Putintseva, E. V. et al. Regulatory $\mathrm{T}$ cells exhibit distinct features in human breast cancer. Immunity. 45, 1122-1134 (2016).

21. Tirosh, I., Izar, B., Prakadan, S. M., Wadsworth, M. H., Treacy, D., Trombetta, J. J. et al. Dissecting the multicellular ecosystem of metastatic melanoma by singlecell RNA-seq. Science 352, 189-196 (2016).

22. Chang, W. H., Forde, D. \& Lai, A. G. A novel signature derived from immunoregulatory and hypoxia genes predicts prognosis in liver and five other cancers. $J$. Transl. Med. 17(Jan), 14 (2019).

23. Chang W. H., Forde D., Lai A. G. Dual prognostic role for 2-oxoglutarate oxygenases in ten diverse cancer types: Implications for cell cycle regulation and cell adhesion maintenance. Cancer Commun. 39, 23 (2019).

24. Chang, W. H. \& Lai, A. G. Timing gone awry: distinct tumour suppressive and oncogenic roles of the circadian clock and crosstalk with hypoxia signalling in diverse malignancies. J. Transl. Med. 17(Apr), 132 (2019).

25. Tabas-Madrid, D., Nogales-Cadenas, R. \& Pascual-Montano, A. GeneCodis3: a nonredundant and modular enrichment analysis tool for functional genomics. Nucleic Acids Res. 40(W1), W478-W483 (2012).

26. Kuleshov, M. V., Jones, M. R., Rouillard, A. D., Fernandez, N. F., Duan, Q., Wang, Z et al. Enrichr: a comprehensive gene set enrichment analysis web server 2016 update. Nucleic Acids Res. 44(W1), W90-W97 (2016).

27. Haider, S., Mclntyre, A., van Stiphout, R. G. P. M., Winchester, L. M., Wigfield, S., Harris, A. L. et al. Genomic alterations underlie a pan-cancer metabolic shift associated with tumour hypoxia. Genome Biol. 17, 1-17 (2016).

28. Chang W. H., Lai A. G. An immunoevasive strategy through clinically-relevant pan-cancer genomic and transcriptomic alterations of JAK-STAT signaling components. bioRxiv [Internet]. 2019; Available from: https://www.biorxiv.org/ content/early/2019/03/14/576645.

29. Chang, W. H. \& Lai, A. G. The pan-cancer mutational landscape of the PPAR pathway reveals universal patterns of dysregulated metabolism and interactions with tumor immunity and hypoxia. Ann. N Y Acad. Sci. 1448(Jul), 65-82 (2019). 
30. Bao, B., Wang, Z., Ali, S., Kong, D., Banerjee, S., Ahmad, A. et al. Over-expression of FoxM1 leads to epithelial-mesenchymal transition and cancer stem cell phenotype in pancreatic cancer cells. J Cell Biochem. 112, 2296-2306 (2011).

31. Zingg, D., Debbache, J., Schaefer, S. M., Tuncer, E., Frommel, S. C., Cheng, P. et al. The epigenetic modifier EZH2 controls melanoma growth and metastasis through silencing of distinct tumour suppressors. Nat. Commun. 6, 6051 (2015).

32. Li, H., Cai, Q., Wu, H., Vathipadiekal, V., Dobbin, Z. C., Li, T. et al. SUZ12 promotes human epithelial ovarian cancer by suppressing apoptosis via silencing HRK. Mol Cancer Res. 10, 1462-1472 (2012).

33. Hovinga, K. E., Shimizu, F., Wang, R., Panagiotakos, G., Van Der Heijden, M., Moayedpardazi, $\mathrm{H}$. et al. Inhibition of notch signaling in glioblastoma targets cancer stem cells via an endothelial cell intermediate. Stem Cells. 28, 1019-1029 (2010).

34. Bussolati, B., Dekel, B., Azzarone, B. \& Camussi, G. Human renal cancer stem cells. Cancer Lett. 338, 141-146 (2013)

35. Suvà, M.-L., Riggi, N., Janiszewska, M., Radovanovic, I., Provero, P., Stehle, J.-C. et al. EZH2 is essential for glioblastoma cancer stem cell maintenance. Cancer Res. 69, 9211-9218 (2009). 8-5472.

36. Sauvageau, M. \& Sauvageau, G. Polycomb group proteins: multi-faceted regulators of somatic stem cells and cancer. Cell Stem Cell. 7, 299-313 (2010).

37. Johnson, R., Teh, C. H., Kunarso, G., Wong, K. Y., Srinivasan, G., Cooper, M. L. et al. REST regulates distinct transcriptional networks in embryonic and neural stem cells. PLoS Biol. 6, e256 (2008).

38. Keith, B. \& Simon, M. C. Hypoxia-inducible factors, stem cells, and cancer. Cell. 129, 465-472 (2007).

39. Gustafsson, M. V., Zheng, X., Pereira, T., Gradin, K., Jin, S., Lundkvist, J. et al. Hypoxia requires notch signaling to maintain the undifferentiated cell state. Dev Cell. 9, 617-628 (2005).

40. Li, Z., Bao, S., Wu, Q., Wang, H., Eyler, C., Sathornsumetee, S. et al. Hypoxiainducible factors regulate tumorigenic capacity of glioma stem cells. Cancer Cell. 15, 501-513 (2009).

41. Bao, S., Wu, Q., Sathornsumetee, S., Hao, Y., Li, Z., Hjelmeland, A. B. et al. Stem cell-like glioma cells promote tumor angiogenesis through vascular endothelial growth factor. Cancer Res. 66, 7843-7848 (2006).

42. Kawasaki, B. T. \& Farrar, W. L. Cancer stem cells, CD200 and immunoevasion. Trends Immunol. 29, 464-468 (2008).

43. Ledaki, I., McIntyre, A., Wigfield, S., Buffa, F., McGowan, S., Baban, D. et al. Carbonic anhydrase IX induction defines a heterogeneous cancer cell response to hypoxia and mediates stem cell-like properties and sensitivity to HDAC inhibition. Oncotarget 6, 19413-19427 (2015).

44. Clambey, E. T., McNamee, E. N., Westrich, J. A., Glover, L. E., Campbell, E. L., Jedlicka, P. et al. Hypoxia-inducible factor-1 alpha-dependent induction of FoxP3 drives regulatory T-cell abundance and function during inflammatory hypoxia of the mucosa. Proc. Natl. Acad. Sci. USA 109, E2784-E2793 (2012).

45. Doedens, A. L., Stockmann, C., Rubinstein, M. P., Liao, D., Zhang, N., DeNardo, D. G. et al. Macrophage expression of hypoxia-inducible factor-1a suppresses T-cell function and promotes tumor progression. Cancer Res. 70, 7465-7475 (2010).

46. Noman, M. Z., Hasmim, M., Messai, Y., Terry, S., Kieda, C., Janji, B. et al. Hypoxia: a key player in antitumor immune response. A Review in the Theme: Cellular Responses to Hypoxia. Am. J. Physiol. Cell. Physiol. 309, C569-C579 (2015).

47. Huang, S., He, J., Zhang, X., Bian, Y., Yang, L., Xie, G. et al. Activation of the hedgehog pathway in human hepatocellular carcinomas. Carcinogenesis. 27, 1334-1340 (2006).

48. Villanueva, A., Alsinet, C., Yanger, K., Hoshida, Y., Zong, Y., Toffanin, S. et al. Notch signaling is activated in human hepatocellular carcinoma and induces tumor formation in mice. Gastroenterology. 143, 1660-1669 (2012).
49. Sjölund, J., Johansson, M., Manna, S., Norin, C., Pietras, A., Beckman, S. et al. Suppression of renal cell carcinoma growth by inhibition of Notch signaling in vitro and in vivo. J Clin Invest. 118, 217-228 (2008).

50. Yeh, T.-S., Wu, C.-W., Hsu, K.-W., Liao, W.-J., Yang, M.-C., Li, A. F.-Y. et al. The activated Notch 1 signal pathway is associated with gastric cancer progression through cyclooxygenase-2. Cancer Res. 69, 5039-5048 (2009).

51. Chang, W. H. \& Lai, A. G. Pan-cancer genomic amplifications underlie a Wnt hyperactivation phenotype associated with stem cell-like features leading to poor prognosis. Transl Res. 208, 47-62 (2019).

52. Palagani, V., Bozko, P., El Khatib, M., Belahmer, H., Giese, N., Sipos, B. et al. Combined inhibition of Notch and JAK/STAT is superior to monotherapies and impairs pancreatic cancer progression. Carcinogenesis. 35, 859-866 (2013).

53. Javelaud, D., Alexaki, V. I., Dennler, S., Mohammad, K. S., Guise, T. A. \& Mauviel, A. TGF- $\beta / S M A D / G L I 2$ signaling axis in cancer progression and metastasis. Cancer Res. 71, 5606-5610 (2011).

54. Kim, E., Kim, M., Woo, D.-H., Shin, Y., Shin, J., Chang, N. et al. Phosphorylation of EZH2 activates STAT3 signaling via STAT3 methylation and promotes tumorigenicity of glioblastoma stem-like cells. Cancer Cell. 23, 839-852 (2013).

55. Sasaki, M., Ikeda, H., Itatsu, K., Yamaguchi, J., Sawada, S., Minato, H. et al. The overexpression of polycomb group proteins $\mathrm{Bmi} 1$ and $\mathrm{EZH} 2$ is associated with the progression and aggressive biological behavior of hepatocellular carcinoma. Lab. Investig. 88, 873 (2008).

56. Cheng, A. S. L., Lau, S. S., Chen, Y., Kondo, Y., Li, M. S., Feng, H. et al. EZH2mediated concordant repression of Wnt antagonists promotes $\beta$-catenin-dependent hepatocarcinogenesis. Cancer Res. 71, 4028-4039 (2011).

57. Wagener, N., Macher-Goeppinger, S., Pritsch, M., Hüsing, J., Hoppe-Seyler, K., Schirmacher, P. et al. Enhancer of zeste homolog 2 (EZH2) expression is an independent prognostic factor in renal cell carcinoma. BMC Cancer. 10, 524 (2010).

58. Zhang, J., Wu, W., Xu, S., Zhang, J., Zhang, J., Yu, Q. et al. MicroRNA-105 inhibits human glioma cell malignancy by directly targeting SUZ12. Tumor Biol. 39, 1010428317705766 (2017)

59. Pan, Q., Li, Q., Liu, S., Ning, N., Zhang, X., Xu, Y. et al. Concise review: targeting cancer stem cells using immunologic approaches. Stem Cells. 33, 2085-2092 (2015).

60. Wu, A., Wiesner, S., Xiao, J., Ericson, K., Chen, W., Hall, W. A. et al. Expression of $\mathrm{MHC}$ I and NK ligands on human CD133+ glioma cells: possible targets of immunotherapy. J Neurooncol. 83, 121-131 (2007).

61. Wang, B., Wang, Q., Wang, Z., Jiang, J., Yu, S.-C., Ping, Y.-F. et al. Metastatic consequences of immune escape from NK cell cytotoxicity by human breast cancer stem cells. Cancer Res. 74, 5746-5757 (2014).

62. Soeda, A., Park, M., Lee, D., Mintz, A., Androutsellis-Theotokis, A., McKay, R. D. et al. Hypoxia promotes expansion of the CD133-positive glioma stem cells through activation of HIF-1a. Oncogene. 28, 3949 (2009)

63. Heddleston, J. M., Li, Z., McLendon, R. E., Hjelmeland, A. B. \& Rich, J. N. The hypoxic microenvironment maintains glioblastoma stem cells and promotes reprogramming towards a cancer stem cell phenotype. Cell cycle. 8, 3274-3284 (2009).

64. Azzi, S., Bruno, S., Giron-Michel, J., Clay, D., Devocelle, A., Croce, M. et al. Differentiation therapy: targeting human renal cancer stem cells with interleukin 15. J. Natl. Cancer Inst. 103, 1884-1898 (2011).

65. Mathieu, J., Zhang, Z., Zhou, W., Wang, A. J., Heddleston, J. M., Pinna, C. M. A. et al. HIF induces human embryonic stem cell markers in cancer cells. Cancer Res. 71, 4640-4652 (2011).

66. Topalian, S. L., Drake, C. G. \& Pardoll, D. M. Immune checkpoint blockade: a common denominator approach to cancer therapy. Cancer Cell. 27, 450-461 (2015). 